\title{
COHERENT STRUCTURES AND CARRIER SHOCKS IN THE NONLINEAR PERIODIC MAXWELL EQUATIONS
}

\author{
GIDEON SIMPSON AND M.I. WEINSTEIN
}

\begin{abstract}
We consider the one-dimensional propagation of electromagnetic waves in a weakly nonlinear and low-contrast spatially inhomogeneous medium with no energy dissipation. We focus on the case of a periodic medium, in which dispersion enters only through the (Floquet-Bloch) spectral band dispersion associated with the periodic structure; chromatic dispersion ( time-nonlocality of the polarization) is neglected. Numerical simulations show that for initial conditions of wave-packet type (a plane wave of fixed carrier frequency multiplied by a slow varying, spatially localized function) very long-lived spatially localized coherent soliton-like structures emerge, whose character is that of a slowly varying envelope of a train of shocks. We call this structure an envelope carrier-shock train.

The structure of the solution violates the oft-assumed nearly monochromatic wave packet structure, whose envelope is governed by the nonlinear coupled mode equations (NLCME). The inconsistency and inaccuracy of NLCME lies in the neglect of all (infinitely many) resonances except for the principle resonance induced by the initial carrier frequency. We derive, via a nonlinear geometrical optics expansion, a system of nonlocal integro-differential equations governing the coupled evolution of backward and forward propagating waves. These equations incorporate effects of all resonances. In a periodic medium, these equations may be expressed as a system of infinitely many coupled mode equations, which we call the extended nonlinear coupled mode system (xNLCME). Truncating xNLCME to include only the principle resonances leads to the classical NLCME.

Numerical simulations of xNLCME demonstrate that it captures both large scale features, related to third harmonic generation, and fine scale carrier shocks features of the nonlinear periodic Maxwell equations.
\end{abstract}

\section{Contents}

1. Overview 1

1.1. Summary of results 4

2. Nonlinear Maxwell and NLCME 7

3. Simulations of nonlinear periodic Maxwell

3.1. Soliton-like coherent structures

3.2. Envelope carrier-shock trains

4. Resonant nonlinear geometrical optics and nonlinear spatially inhomogeneous Maxwell equations 14

4.1. Nonlinear geometric optics expansion

4.2. Periodic Media and xNLCME

4.3. Conservation Laws and Hamiltonian Structure 17

5. Simulations of the Truncated xNLCME

6. Summary and discussion 21

References $\quad[23$

Appendix A. The NLCME Soliton

Appendix B. Simulating the Nonlinear Maxwell Equations 26

B.1. Finite Volume Methods for Maxwell

B.2. Non-Convex Fluxes and the Entropy Condition 27

\section{Overview}

Realized and potential applications of microstructured dielectric media motivate a thorough mathematical study of wave-propagation governed by nonlinear hyperbolic equations, e.g. Maxwell's equations with 
periodic and nonlinear constituitive laws. This paper explores a class of nonlinear hyperbolic equations with a spatially periodic flux function:

$$
\begin{aligned}
\partial_{t} \mathbf{v}+\partial_{x} \mathbf{f}(x, \mathbf{v}) & =\mathbf{0} \\
\mathbf{f}(x, \mathbf{0}) & =\mathbf{0}, \\
\mathbf{f}(x+2 \pi, \mathbf{v}) & =\mathbf{f}(x, \mathbf{v}) .
\end{aligned}
$$

In particular, we shall assume that periodic variations are weak (a low contrast structure) and study solutions, whose amplitude is small and such that the effects of periodicity-induced dispersion and nonlinearity are in balance.

Indeed, a non-trivial spatially periodic structure is dispersive. This can be seen by linearizing (1.1) about the state $\mathbf{v}=\mathbf{0}$, giving the linear system:

$$
\partial_{t} \mathbf{V}+\partial_{x}\left(D_{\mathbf{v}} \mathbf{f}(x, \mathbf{0}) \mathbf{V}\right)=\mathbf{0},
$$

which retains periodicity. Floquet-Bloch theory $7 \sqrt{38}$ implies that associated to the PDE $(1.2)$ is a family of band dispersion functions $k \mapsto \omega_{j}(k), k \in\left(-\frac{1}{2}, \frac{1}{2}\right]$. Wave propagation is dispersive since the group velocities, $\omega_{j}^{\prime}(k)$, are typically non-zero. Thus, waves of different wavelengths travel with different speeds.

Dispersive properties, encoded in the functions $\omega_{j}(\cdot)$ and the associated Floquet-Bloch states, can be manipulated by tuning the periodic structure through, for example, modification of the periodic lattice, the maximum and minimum variations of $D_{\mathbf{v}} \mathbf{f}(x, \mathbf{0})$ (material contrast), etc.

It is well-known that for general initial conditions, solutions of hyperbolic systems of conservation laws with spatially homogeneous nonlinear fluxes:

$$
\partial_{t} \mathbf{v}+\partial_{x} \mathbf{f}(\mathbf{v})=\mathbf{0}
$$

develop singularities (shocks) in finite time, 20,22 .

Question 1: Is spectral band dispersion, due to a periodic structure, sufficient to arrest shock formation? 1

The ability to control or inhibit the formation of singularities in nonlinear wave propagation could have significant impact in, for example, electromagnetics and elasticity. Strictly speaking, the answer to Question 1 is no. Indeed, for a system of the form (1.1), let us suppose that the flux function was periodically piecewise constant. Finite propagation speed considerations imply that for appropriate initial data, which are sufficiently localized within a uniform region, a shock will form. The dispersive character of the periodic structure is manifested only on sufficiently large spatial and temporal scales. Thus, the problem of controlling shock formation should be posed relative to some class of initial conditions.

A second motivation is the study and design of media which support the propagation of stable soliton-like pulses. These have applications to optical devices which transfer store or, in general, process information which is encoded as light pulses. Associated with dispersive wave-propagation at wavenumber $k_{\star}$ is a dispersion length $\sim\left(\omega^{\prime \prime}\left(k_{\star}\right)\right)^{-1}$. Soliton formation is possible on length scales where the dispersion length and the characteristic length on which nonlinear effects act are comparable. Technological advances have made it possible to fabricate microstructured media with specified dispersion lengths at specified wavelengths. For a given dispersion length, a balance between dispersion and nonlinearity is achieved by tuning the strength of the nonlinear effects through adjusting the field intensity (by an amount which is material dependent). An example of this balance at work is gap soliton formation in periodic structures. These are experiments in optical fiber periodic structures (gratings) involving highly intense (nonlinear) light with carrier wave-length satisfying the Bragg (resonance) condition. The length-scale of such solitons is $10^{-2}$ meters [8].

Theory predicts the existence of gap solitons traveling at any speed, $v$, between zero and the speed of light, $c$ 1] 3. Experiments 8 demonstrate speeds as low as .3c to $.5 c$. Potential applications of gap solitons, based on the design of appropriate localized defects in a periodic structure, are all-optical storage devices [10].

\footnotetext{
${ }^{1}$ For example, though typical smooth initial data for the inviscid Burgers equation $\partial_{t} u+u \partial_{x} u=0$ develop shocks in finite time, the corresponding solutions of the Korteweg - de Vries (KdV) equation, $\partial_{t} u+u \partial_{x} u+\partial_{x}^{3} u=0$, a dispersive perturbation, remain smooth for all time.
} 

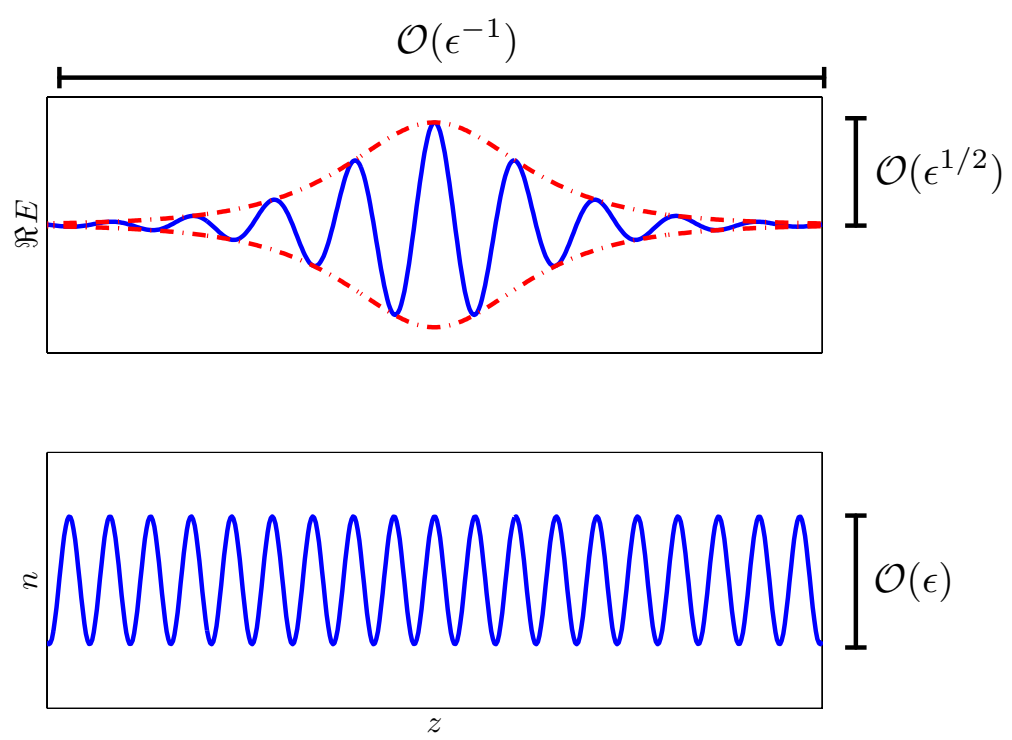

Figure 1. A wavepacket (real part, $\Re E_{0}(z)$, of complex field) with carrier wave length equal twice that of the waveguide refractive index $(n(z))$.

The term gap soliton is used due to frequency of the gap soliton envelope lying in the spectral gap of the linearized system.

Physical predictions of gap solitons are based on explicit solutions of nonlinear coupled mode equations (NLCME), given below in (2.9). NLCME has been formally derived in, for example, [4] from (1.4); see also the discussion in Section 2, Rigorous derivations of NLCME, from models with appropriate dispersion have been presented for the anharmonic Maxwell-Lorentz equations [11] and other nonlinear dispersive equations; see e.g. $12,34,35,40,41$.

Within the approximation of a small amplitude wave field as a wave-packet with slowly varying envelope and single carrier frequency, propagating through a low contrast periodic structure near the Bragg resonance ( see scaling in Figure 1) ), NLCME is argued to govern the principle forward and backward slowly varying envelopes of carrier waves; see [4] and references therein.

As discussed in [11] and in section 2, if the only source of dispersion is the spatial dispersion of the periodic medium (e.g. negligible chromatic dispersion) for weakly nonlinear waves in low contrast media all nonlinearity-generated harmonics are resonant and therefore all mode amplitudes are coupled at leading order. The correct mathematical description would appear to require infinitely many interacting modes. Thus, the classical NLCME are not a mathematically consistent approximation. NLCME may however be satisfactory physical description, for some purposes.t Indeed, the soliton wave form prediction based on NLCME appears to describe some features of experiment.

Question 2: Do nonlinear periodic hyperbolic systems have stable coherent structures, and can one develop a mathematical theory? How are the classical NLCME related to this theory? See the discussion in section 6.

\footnotetext{
${ }^{2}$ Physicists argue in two ways that the coupling to higher harmonics is argued to be negligible : (i) The material systems considered are dissipative at higher wave numbers. Higher wave numbers are damped and therefore these mode amplitudes can be ignored, and (ii) Chromatic dispersion (arising due to the finite time response of the medium to the field) causes nonlinearly generated harmonics to be off-resonance. Therefore, an initial condition exciting the principle modes will not appreciable excite higher harmonics. These rationales are somewhat ad hoc since the precise damping mechanisms are not well-understood and chromatic dispersion is a much weaker effect than photonic band dispersion for weak periodic structures.
} 


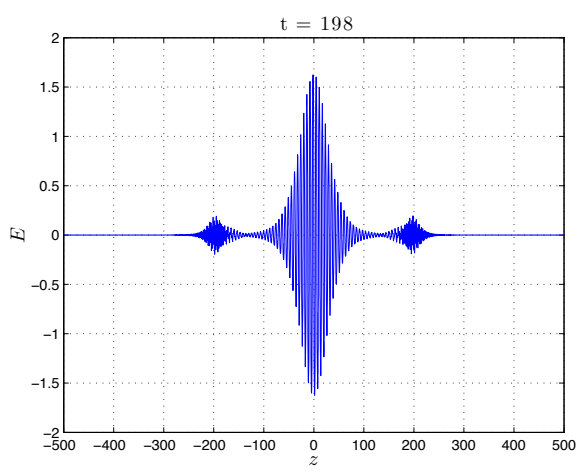

(a)

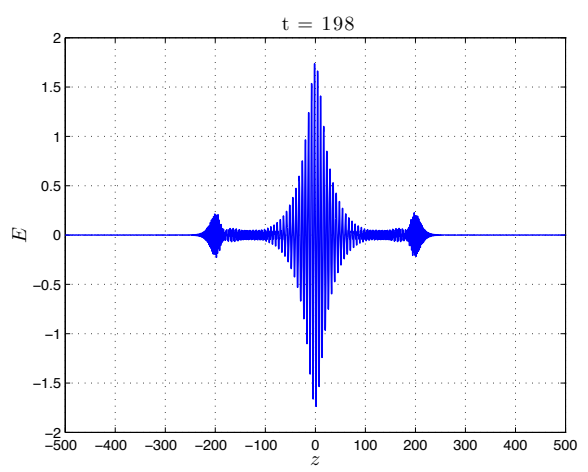

(b)

Figure 2. On the left is a simulation of the Maxwell equations. On the right is the simulation of a truncated asymptotic system, resolving the first and third harmonics. Both simulations were initiated with the same initial conditions. The two side pulses about the main wave appear to be the result of third harmonic generation.

In this article we report on progress on Questions 1 and 2 in the context of the one-dimensional, nonlinear Maxwell equations governing the electric $(E)$ and magnetic $(B)$ fields:

$$
\begin{aligned}
& \partial_{t} D=\partial_{z} B, \\
& \partial_{t} B=\partial_{z} E .
\end{aligned}
$$

with constitutive law

$$
\begin{aligned}
D & =\epsilon(z, E) E \equiv\left(n^{2}(z)+\chi E^{2}\right) E \\
n(z) & =n_{0}+\epsilon N(z)
\end{aligned}
$$

$n(z)$ is a linear refractive index, consisting of a nonzero background average part, $n_{0}$, and a fluctuating (e.g. periodic) part $\epsilon N(z)$. The nonlinear term $\chi E^{2}$ is the nonlinear refractive index, arising from the Kerr effect; in regions of high intensity the refractive index is higher. The consituitive law (1.5), prescribes $D$ as a a local function of $E$. Thus chromatic dispersion, which arises due a time-nonlocal relation between $D$ and $E$ has been neglected. For simplicity, we assume $n_{0}=1$, which can be arranged by a simple scaling.

\subsection{Summary of results.}

(1) In section 3 we present numerical simulations of the nonlinear periodic Maxwell equations, (1.4), for initial data obtained from the explicit NLCME soliton. Under this time-evolution there is robust spatially localized structure on the scale of the NLCME soliton envelope. The persistence of a localized structure and speed of propagation are consistent with that of the NLCME soliton. There is, however, a deviation from the NLCME soliton related to third harmonic generation; these are the two accessory pulses around the principle wave in Figure 2 (a).

(2) On the microscopic scale of the carrier there is nonlinear steepening and shock formation. Therefore, the solution does not evolve as a slowly varying envelope of a single frequency carrier wave. The longlived and spatially localized coherent structure which emerges has the character of a slowly varying envelope of a train of shocks. We call this an envelope carrier-shock train. Figure 3 illustrates the shock-like small spatial scale behavior under slowly varying envelope.

(3) Numerical solution of the nonlinear Maxwell equation (1.4) is non-trivial due to the cubic nonlinearity. As a hyperbolic system, it is neither genuinely nonlinear nor linearly degenerate 23, 29, 44 . To solve by finite volume methods, as we do, an explicit solution of the Riemann problem must be constructed. Details of this are given in Appendix B.

The appropriate entropy condition could, in principle be derived from physical regularization mechanisms, which play the role of viscosity in gas dynamics. However, these mechanisms are not 


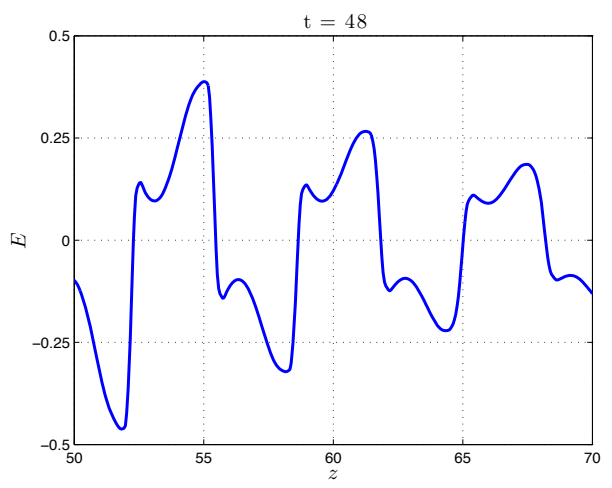

(a)

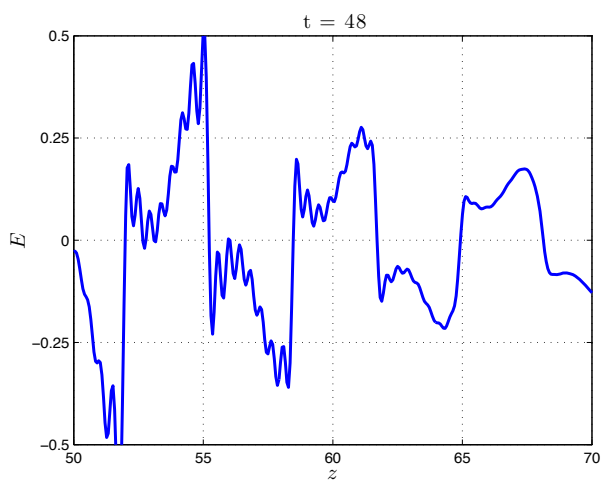

(b)

Figure 3. On the left is a simulation of the Maxwell equations. On the right is the simulation of a truncated asymptotic system. Both simulations were initiated with the same initial conditions. There is an indication of shock formation in the left. On the right, we see that once sufficiently many harmonics are included, the Gibbs effect appears, confirming shock formation.

well understood. However, such mechanisms and the appropriate notion of weak solution would respect thermodynamic principles, which are built into our numerical scheme.

(4) Using a nonlinear geometric optics expansion $6,13,16,21,30$, systematically keeping all resonances, we obtain nonlocal equations governing the interaction of all forward and backward propagating modes. Our asymptotic nonlocal system captures the slowly varying envelope of carrier-shock structures described above; see below.

Specifically, we introduce the general wave-form (much more general than a slowly varying envelope of a nearly monochromatic carrier plane wave), which includes all harmonics

$$
E(z, t)=\epsilon^{1 / 2}\left(E^{+}(z-t, \epsilon z, \epsilon t)+E^{-}(z+t, \epsilon z, \epsilon t)+\mathcal{O}(\epsilon)\right) .
$$

Let

$$
\phi_{ \pm}=z \mp t, \quad \epsilon t=T, \text { and } \epsilon z=Z .
$$

At leading order, the slow evolution of backward and forward components is governed by the coupled integro-differential equations:

$$
\begin{aligned}
\partial_{T} E^{+}+\partial_{Z} E^{+}= & \partial_{\phi}\left\langle N\left(\phi_{+}+s\right) E^{-}\left(\phi_{+}+2 s, Z, T\right)\right\rangle_{s} \\
& +\Gamma \partial_{\phi}\left[\frac{1}{3}\left(E^{+}\right)^{3}+E^{+}\left\langle\left(E^{-}\right)^{2}\right\rangle\right], \\
\partial_{T} E^{-}-\partial_{Z} E^{-}= & -\partial_{\phi}\left\langle N\left(\phi_{-}-s\right) E^{+}\left(\phi_{-}-2 s, Z, T\right)\right\rangle_{s} \\
& -\Gamma \partial_{\phi}\left[\left\langle\left(E^{+}\right)^{2}\right\rangle E^{-}+\frac{1}{3}\left(E^{-}\right)^{3}\right] .
\end{aligned}
$$

Here $\langle\cdot\rangle$ is an averaging operation in the $\phi$ argument;

$$
\langle f\rangle \equiv \lim _{T \rightarrow \infty} \frac{1}{T} \int_{0}^{T} f(s) d s ;
$$

see also section 4. Equations (1.8) arise as a constraint on $E^{ \pm}\left(\phi_{ \pm}, Z, T\right)$ ensuring that the $\mathcal{O}(\epsilon)$ error term 1.7 in remains small on large time scales: $T=\mathcal{O}(1)$ or equivalently $t=\mathcal{O}\left(\epsilon^{-1}\right)$.

Spatial variations in the refractive index, $N(z)$, give rise to a coupling of backward and forward waves. Indeed, if $N(z) \equiv 0$ and one specifies data for the system 1.8$)$ at $t=0$ with non-zero forward components $\left(E^{+} \neq 0\right)$ and, no backward components $\left(E^{-}=0\right)$ then, formally, $E^{-}$remains zero for 


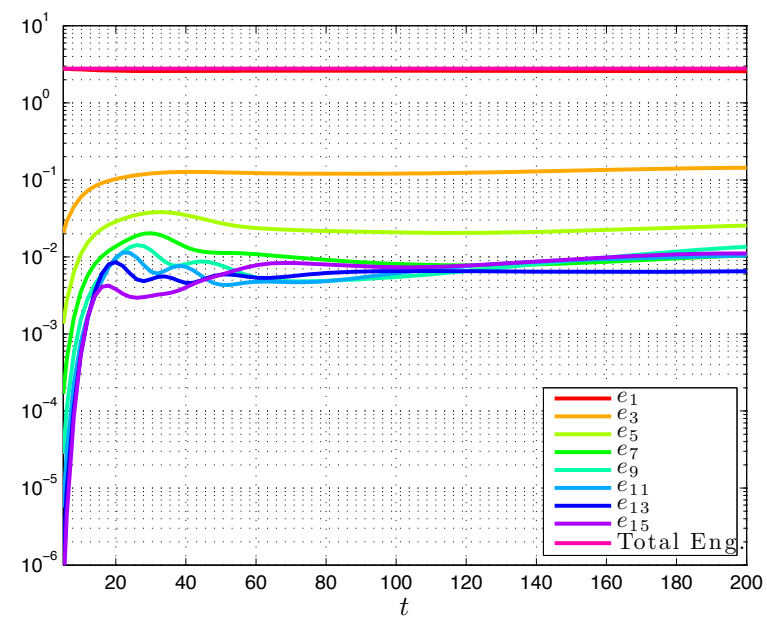

Figure 4. Truncating 1.10 to odd harmonics $|p| \leq 16$, we simulate the initial value problem an NLCME soliton in the first harmonic, and the others zero. The above time series of the energy associated with each harmonic, $e_{p}$, shows that most of the energy continues to reside in the first harmonic.

all time, i.e. no backward waves are generated. Continuing with this assumption of $N=0$ and $E_{0}^{-}=0$, if we let $V(\phi, T)=E^{+}\left(\phi, Z_{0}-T, T\right)$, with $Z_{0}$ arbitrary, then $V$ satisfies

$$
\partial_{T} V=\frac{\Gamma}{3} \partial_{\phi}\left(V^{3}\right) .
$$

This generalized Burger's equation will gives rise to a finite time singularity. We revisit this observation in the discussion, section 6 , when considering how singularities might appear when the linear coupling between backwards and forwards waves is restored, $N \neq 0$.

(5) The nonlocal equations may also be written as an infinite system of coupled mode equations. In the case where $E^{ \pm}$is $2 \pi$ - periodic in $\phi_{ \pm}$, the integro-differential equation 1.8 reduces to an infinite system of coupled mode equations for the Fourier coefficients $\left\{E_{p}^{ \pm}(Z, T): p \in \mathbb{Z}\right\}$ :

$$
\begin{aligned}
\partial_{T} E_{p}^{+}+\partial_{Z} E_{p}^{+}=\mathrm{i} p N_{2 p} E_{p}^{-}+\mathrm{i} p \frac{\Gamma}{3}\left[\sum E_{q}^{+} E_{r}^{+} E_{p-q-r}^{+}\right. \\
\left.+3\left(\sum\left|E_{q}^{-}\right|^{2}\right) E_{p}^{+}\right], \\
\partial_{T} E_{p}^{-}-\partial_{Z} E_{p}^{-=}=\mathrm{i} p \bar{N}_{2 p} E_{p}^{+}+\mathrm{i} p \frac{\Gamma}{3}\left[\sum E_{q}^{-} E_{r}^{-} E_{p-q-r}^{-}\right. \\
\left.+3\left(\sum\left|E_{q}^{+}\right|^{2}\right) E_{p}^{-}\right] .
\end{aligned}
$$

We call this system the extended nonlinear coupled mode equations (xNLCME). xNLCME reduces to the classical NLCME if we neglect higher harmonics.

(6) Simulations of successively higher dimensional mode truncations of 1.10 show improved resolution of the carrier shocks under a slowly varying envelope, whose scale is captured by a comparatively low order truncation. Indeed, Figure 2 (b) shows that inclusion of the third harmonic in the asymptotic system resolves the large scale feature, while inclusion of additional harmonics in Figure 3 (b) shows the Gibbs effect, expected for a finite Fourier representation of a discontinuous function. This demonstrates that our asymptotic analysis leads to equations capturing the essential features of nonlinear Maxwell. However, if we consider how energy, initially only in the first harmonic, is redistributed in time, we see in Figure 4 that most of the energy persists in the first harmonic. This reflects the partial success of NLCME as a model for periodic nonlinear Maxwell.

are

Relation to previous work: Some of the earliest examinations on optical shocks can be found in Rosen, [39], and, DeMartini et al. [5]. In these works, the authors applied the method of characteristics to a unidicretional 
model. Kinsler and Kinsler et al. have continued to examine this problem, and have developed an algorithm for detecting the onset of shock formation, 18,19. Carrier shocks were also examined by Flesch, Moloney, \& Mlejnek, [9], for spatially homogeneous Maxwell system with chromatic dispersion, modeled via a timenonlocal Lorentzian polarization response. Ranka, Windeler, \& Stentz have found experimental evidence of optical shocks, [37. In their work, a monochromatic pulse with sufficient power steepened and generated a broadband optical continuum.

Coherent structures in nonlinear and periodic media have also been studied by LeVeque, LeVeque \& Yong, and Ketcheson [17, 25, 26] in a model for heterogeneous nonlinear elastic media. They considered order one solutions in high contrast, rapidly varying, periodic structures. Their simulations yielded localized structures on the scale of many periods with oscillations on the scale of the period. For piecewise constant (discontinuous) periodic structures, they have a discontinuous carrier shock-like character on the scale of the period, though this is due to discontinuities in the medium, the fluxes remain continuous. A twoscale (homogenization) expansion yields a nonlinear dispersive equation, with solitary waves, similar to the computed solution envelope. In their physical regime, the variations in the properties of the media and the nonlinearity are $\mathcal{O}(1)$. In contrast, we consider an asymptotic regime where the constrast of the periodic structure and nonlinearity are of the same order, $\mathcal{O}(\epsilon)$. Furthermore, the initial condition has two scales (envelope and carrier scales), where the carrier wave length is of the same order, indeed in resonance with, the periodic structure. These different scalings lead to different asymptotic descriptions. An early example of the interactions between nonlinearity and a periodic structure was in atmospheric science, studied by Majda et al., 31. In this work, a model of the interaction of equatorial waves with topography gives rise to nonsmooth profiles (in this case, solitary waves with corner singularities).

Finally, systems of coupled modes have also been examined in prior works, though the work is typically limited two just two harmonics, such as a first and second harmonic system or a first and third harmonic system. Such a system was studied by Tasgal, Band, \& Malomed [46], who were able to find stable polychromatic solitons in a first and third harmonic system.

An outline of this paper is as follows. In Section 2, we review how NLCME arises as an approximation of nonlinear Maxwell. Results of Maxwell Simulations, showing the coherent structures and shocks, are given presented in Section 3. We then present our derivation of xNLCME in Section 4. followed by simulations of this system in Section 5. We discuss all of these results in Section 6 .

Acknowledgements: The authors would like to thank R.R. Rosales for discussions during the early stages of this work on the use nonlinear geometrical optics. We also thank M. Pugh, D. Ketcheson, R.J. LeVeque, and C. Sulem for helpful discussions. GS was supported in part by NSF-IGERT grant DGE-0221041, NSF-CMG grant DMS-05-30853, and NSERC. MIW was supported in part by NSF grants DMS-0707850 and DMS-10-08855. MIW would also like to acknowledge the hospitality of the Courant Institute of Mathematical Sciences, where he was on sabbatical during the preparation of this article.

\section{Nonlinear Maxwell and NLCME}

In this section we briefly review how NLCME arises from nonlinear Maxwell with a periodically varying index of refraction. We also identify the mathematical inconsistency of NLCME as a description of the wave-envelope.

First, we write the nonlinear Maxwell equation (1.4) as

$$
\partial_{t}^{2}\left(n(z)^{2} E+\chi E^{3}\right)=\partial_{z}^{2} E
$$

with index of refraction

$$
n(z)=1+\epsilon N(z), \quad 0<\epsilon \ll 1,
$$

where $N(z)$ is $2 \pi$ periodic with mean zero and Fourier series:

$$
N(z)=\sum_{p \in \mathbb{Z} \backslash\{0\}} N_{p} e^{\mathrm{i} p z} .
$$

We shall seek solutions which incorporate (i) slow variations in time and space, due to the weak modulation about a constant refractive index; (ii) a scaling of the wave-field which seeks solutions in which the effects 
of dispersion and nonlinearity are in balance:

$$
E^{\epsilon}(z, t)=\epsilon^{\frac{1}{2}} \mathcal{E}^{\epsilon}(z, t ; Z, T), \quad Z=\epsilon z, \quad T=\epsilon t .
$$

Rewriting (2.1) in terms of new variables dependent $\mathcal{E}^{\epsilon}$ and independent $(z, t, Z, T)$ variables, we obtain:

$$
\left(\partial_{t}^{2}-\partial_{z}^{2}\right) \mathcal{E}^{\epsilon}+\epsilon\left(2 \partial_{t} \partial_{T} \mathcal{E}^{\epsilon}-2 \partial_{z} \partial_{Z} \mathcal{E}^{\epsilon}+2 N(z) \mathcal{E}^{\epsilon}+\chi\left(\mathcal{E}^{\epsilon}\right)^{3}\right)+\mathcal{O}\left(\epsilon^{3}\right)=0 .
$$

Formally expanding $\mathcal{E}^{\epsilon}$ as

$$
\mathcal{E}^{\epsilon}(z, t, Z, T)=\mathcal{E}_{0}(z, t, Z, T)+\epsilon \mathcal{E}_{1}(z, t, Z, T)+\ldots
$$

we obtain the following hierarchy for $\mathcal{E}_{j}(z, t, Z, T), j \geq 0$ :

$$
\begin{aligned}
& \mathcal{O}\left(\epsilon^{0}\right)\left(\partial_{t}^{2}-\partial_{z}^{2}\right) \mathcal{E}_{0}=0 \\
& \mathcal{O}\left(\epsilon^{1}\right)\left(\partial_{t}^{2}-\partial_{z}^{2}\right) \mathcal{E}_{1}=-2 \partial_{t} \partial_{T} \mathcal{E}_{0}+2 \partial_{z} \partial_{Z} \mathcal{E}_{0}-2 N(z) \mathcal{E}_{0}-\chi\left(\mathcal{E}_{0}\right)^{3} \\
& \quad \vdots \\
& \mathcal{O}\left(\epsilon^{j}\right)\left(\partial_{t}^{2}-\partial_{z}^{2}\right) \mathcal{E}_{j}=\text { expressions in terms of } \mathcal{E}_{l}, \quad 0 \leq l \leq j-1
\end{aligned}
$$

Solving the $\mathcal{O}\left(\epsilon^{0}\right)$ equation yields:

$$
\mathcal{E}_{0}(z, t, Z, T)=\mathcal{E}^{+}(Z, T) e^{i(z-t)}+\mathcal{E}^{-}(Z, T) e^{-i(z+t)}+\text { c.c. }
$$

Thus, the leading order consists of backward and forward propagating waves, modulated by the slow envelope amplitude functions $\mathcal{E}^{ \pm}(Z, T)$, which are to be determined.

Substitution of 2.6 into the $\mathcal{O}\left(\epsilon^{1}\right)$ equation for $\mathcal{E}_{1}$ yields the equation:

$$
\begin{aligned}
& \left(\partial_{t}^{2}-\partial_{z}^{2}\right) \mathcal{E}_{1} \\
& =\left[2 i \partial_{T} \mathcal{E}^{+}-2 i \partial_{Z} \mathcal{E}^{+}-2 N_{2} \mathcal{E}^{-}-3 \chi\left(\left|\mathcal{E}^{+}\right|^{2}+2\left|\mathcal{E}^{-}\right|^{2}\right) \mathcal{E}^{+}\right] e^{i(z-t)} \\
& +\left[2 i \partial_{T} \mathcal{E}^{-}-2 i \partial_{Z} \mathcal{E}^{+}-2 \bar{N}_{2} \mathcal{E}^{+}-3 \chi\left(\left|\mathcal{E}^{-}\right|^{2}+2\left|\mathcal{E}^{+}\right|^{2}\right) \mathcal{E}^{-}\right] e^{-i(z+t)} \\
& +\left(\mathcal{E}^{+}\right)^{3} e^{3 i(z-t)}+\left(\mathcal{E}^{-}\right)^{3} e^{-3 i(z+t)}+\text { c.c. }+ \text { non-resonant terms }
\end{aligned}
$$

We have used that $N_{0}=0$ and

$$
\begin{aligned}
& N(z)\left(\mathcal{E}^{+} e^{i(z-t)}+\mathcal{E}^{-} e^{-i(z+t)}\right) \\
& \quad=N_{-2} \mathcal{E}^{+} e^{-i(z+t)}+N_{2} \mathcal{E}^{-} e^{i(z-t)}+\text { c.c. }+ \text { non-resonant terms. }
\end{aligned}
$$

Each term, explicitly written on the right hand side of (2.7), is resonant with the kernel of $\left(\partial_{t}^{2}-\partial_{z}^{2}\right)$. It follows that the coefficients of all harmonic plane waves: $e^{ \pm i q(z-t)}$ and $e^{ \pm i q(z+t)}, q \in \mathbb{Z}$ must vanish for $\mathcal{E}_{1}$ to be bounded in $t$.

The vanishing of the coefficients of $e^{i(z-t)}$ and $e^{-i(z+t)}$ yields the nonlinear coupled mode equations (NLCME):

$$
\begin{aligned}
& \partial_{T} \mathcal{E}^{+}+\partial_{Z} \mathcal{E}^{+}=i N_{2} \mathcal{E}^{-}+i \Gamma\left(\left|\mathcal{E}^{+}\right|^{2}+2\left|\mathcal{E}^{-}\right|^{2}\right) \mathcal{E}^{+}, \\
& \partial_{T} \mathcal{E}^{-}-\partial_{Z} \mathcal{E}^{-}=i \bar{N}_{2} \mathcal{E}^{+}+i \Gamma\left(\left|\mathcal{E}^{-}\right|^{2}+2\left|\mathcal{E}^{+}\right|^{2}\right) \mathcal{E}^{-},
\end{aligned}
$$

where $\Gamma \equiv \frac{3}{2} \chi$ and $\bar{N}_{2}=N_{-2}$. The initial value problem for (2.9) is well-posed 11]. NLCME also has explicit family of gap-soliton solutions; see Appendix A.

However, requiring $\mathcal{E}^{ \pm}$to satisfy $(2.9)$ removes only the lowest harmonic resonances. This is the approximation invoked in the physics literature; see the survey [4] and references cited therein.

Note however that the remaining explicitly displayed terms on the right hand side of (2.7) are resonant as well and induce linear in time growth. If we choose to remove the resonant terms proportional to $e^{3 i(z-t)}$ and $e^{-3 i(z+t)}$ by including slow modulations of these plane waves at $\mathcal{O}\left(\epsilon^{0}\right)$, nonlinearity and parametric forcing through $N(z)$ will generate yet other resonant harmonics. 
A leading order solution which does not generate resonant terms at higher order must contain all harmonics. Thus, NLCME is mathematically inconsistent. In section 4 we derive an integro-differential equation, which consistently incorporates all resonances. As seen from our numerical and asymptotic studies, this nonlocal nonlinear geometrical optics equation more accurately capture features on both small and large spatial scales, e.g. changes in the envelope due to higher harmonic generation, as well as carrier shock formation.

\section{Simulations of nOnlinear Periodic MaXwell}

In this section we discuss the results of numerical simulations, based on the algorithms of Appendix $B$, of the nonlinear and periodic Maxwell equations 2.1.

- In section 3.1 we show that for Cauchy initial data derived from the classical NLCME soliton, there evolve spatially localized soliton-like states which persist on long time scales. We discuss aspects of the large scale (envelope) structure of such states, which are consistent with the NLCME soliton, as well as significant deviations.

- In section 3.2 we show that smoothness breaks down in finite time. In particular, we observe shock formation on the fast spatial scale of the carrier wave, while a slowly varying envelope evolves smoothly.

We begin by expressing (2.1) as a first order system:

$$
\partial_{t}\left(\begin{array}{c}
n(z)^{2} E+\chi E^{3} \\
B
\end{array}\right)+\partial_{z}\left(\begin{array}{c}
-B \\
-E
\end{array}\right)=0 .
$$

We introduce the scaling $(E, B, D)^{T}=\epsilon^{1 / 2}(\tilde{E}, \tilde{B}, \tilde{D})$, and expressing the equations in terms of the variables: $(\tilde{D}, \tilde{B})$ coordinates. Dropping tildes, this is

$$
\partial_{t}\left(\begin{array}{l}
D \\
B
\end{array}\right)+\partial_{z}\left(\begin{array}{c}
-B \\
-E(D, z)
\end{array}\right)=0
$$

where $E(D, z)$ is the unique real solution of

$$
D=n(z)^{2} E+\epsilon \chi E^{3}
$$

3.1. Soliton-like coherent structures. As is well known [1,3] NLCME has spatially localized gap soliton solutions. We use the analytical expression for the gap soliton to generate Cauchy initial data, $E(z, 0), \partial_{t} E(z, 0)$ for 2.1) and numerically simulate the evolution.

Using (2.6) and the leading order approximation for the magnetic field $B_{1}^{ \pm}=\mp E_{1}^{ \pm}$, NLCME soliton data (see A.1 in Appendix A can be seeded into Maxwell using

$$
\begin{aligned}
& E=\mathcal{E}^{+}(\epsilon z, \epsilon t) e^{i(z-t)}+\mathcal{E}^{-}(\epsilon z, \epsilon t) e^{-i(z+t)}+\text { c.c. }, \\
& B=-\mathcal{E}^{+}(\epsilon z, \epsilon t) e^{i(z-t)}+\mathcal{E}^{+}(\epsilon z, \epsilon t) e^{i(z-t)}+\text { c.c. }
\end{aligned}
$$

We obtain $D$ via 3.3 and evaluate at $t=0$ to get the initial condition.

For a spatially varying index of refraction, we take

$$
N(z)=\frac{4}{\pi} \cos (2 z), \quad \text { i.e. } N_{2}=N_{-2}=\frac{2}{\pi}, \quad N_{p}=0, \quad|p| \neq 2,
$$

$\epsilon=0.0625$, and $\chi=1\left(\Gamma=\frac{3}{2}\right)$. The results of our simulations appear in a - d of Figures 5 and 6 . While there is attenuation in amplitude and some dispersive spreading of energy, the solution remains spatially localized over long time intervals. Not only is there a persistence of the localization (with the periodic medium), but also there is good pointwise agreement with the NLCME approximation; see Figure 7.

Frames e - $\mathrm{h}$ of Figures 5 and 6 display the corresponding results in the absence of a periodic structure, i.e. $N(z) \equiv 0$. The delocalization, dispersive spreading and attenuation of the wave amplitude is greatly enhanced. To understand this heuristically, note that a gap soliton is a localized state whose frequency lies in the spectral gap of the linearized PDE at the zero solution. A focusing nonlinearity adds a (self-consistent) potential well, creating a (nonlinear) defect mode with frequency lying in this spectral gap. If $N(z) \equiv 0$ then the linearization at the zero state has no spectral gap. Thus, a oscillating with the gap soliton frequency would couple to radiation modes and dispersively spread and attenuate. This mechanism is discussed, for example, in 45 . 
Simulations with varying refractive index, 3.5 :

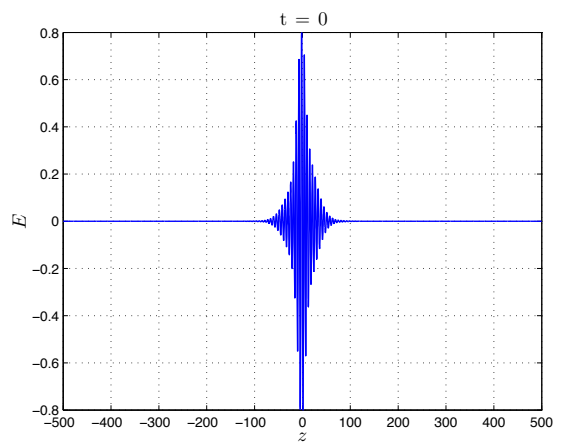

(a)

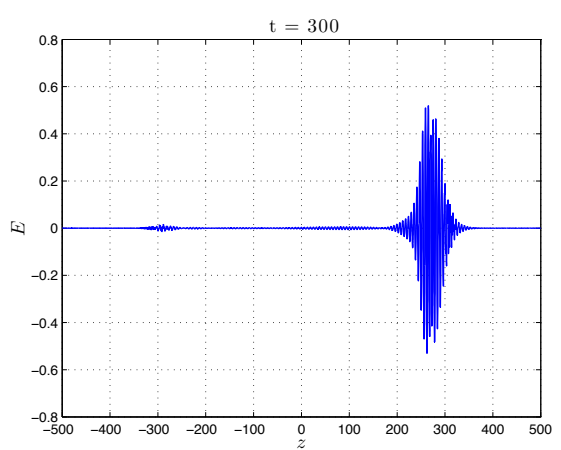

(c)

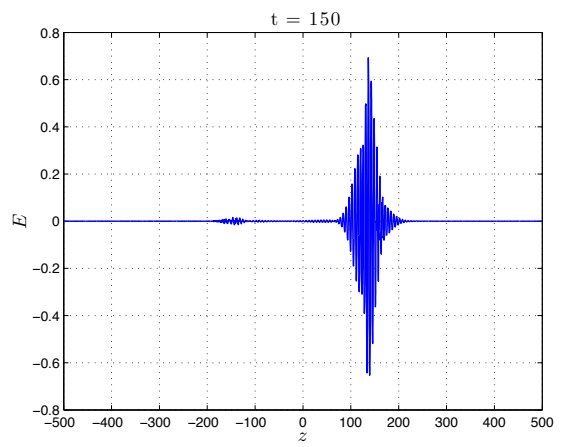

(b)

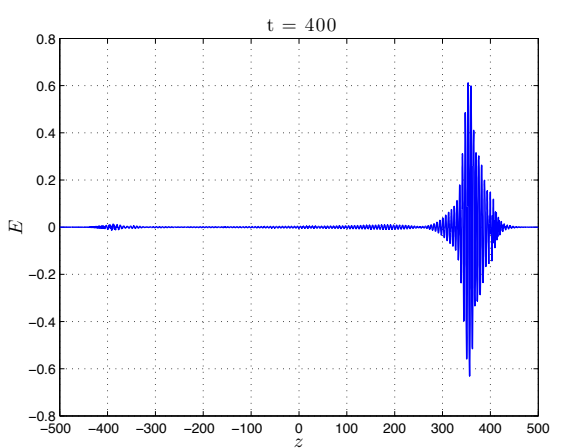

(d)

Simulations with constant refractive index, $N(z)=0$ :

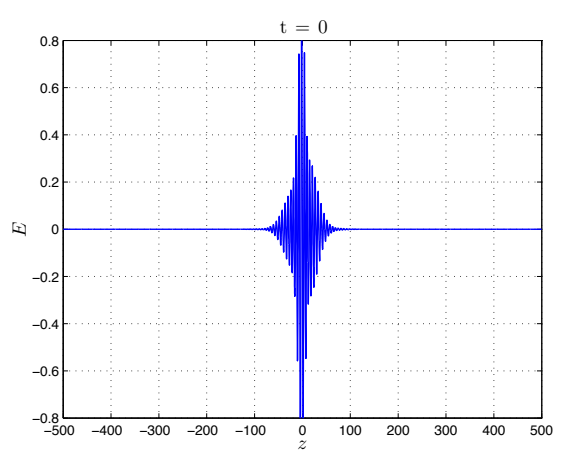

(e)

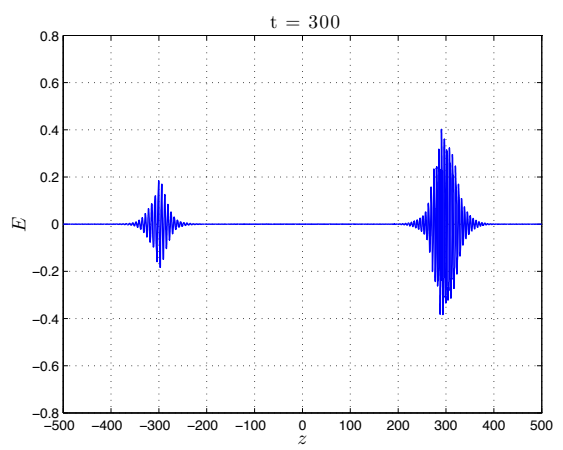

(g)

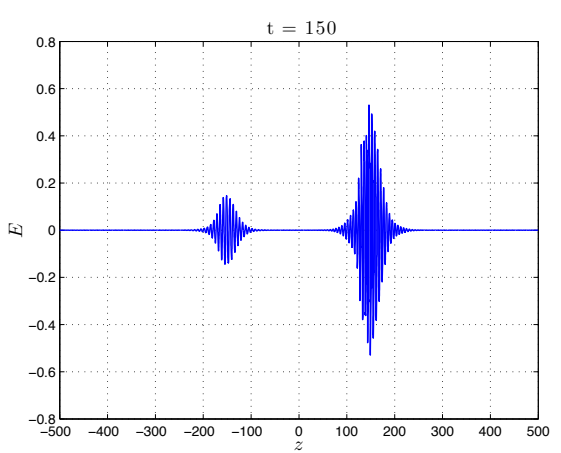

(f)

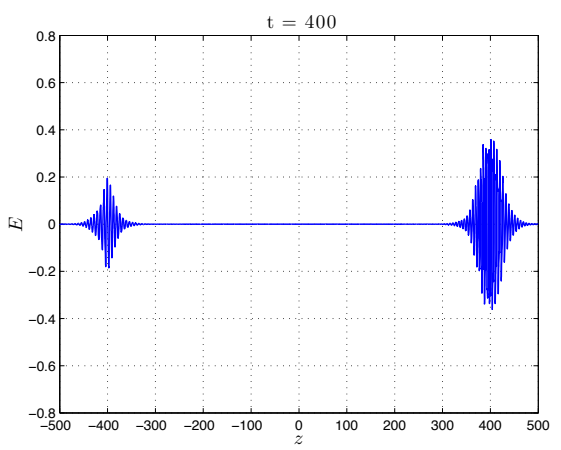

(h)

Figure 5. Rescaled Maxwell equation, (3.29), time-evolution for data generated by the NLCME soliton with parameters $v=.9$ and $\delta=.9$; see A.1). The solutions are computed with 20000 grid points on the domain $[-500,500]$. 
Simulations with varying refractive index, 3.5 :

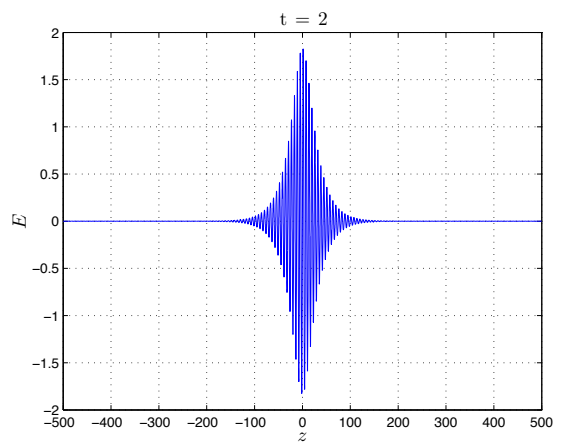

(a)

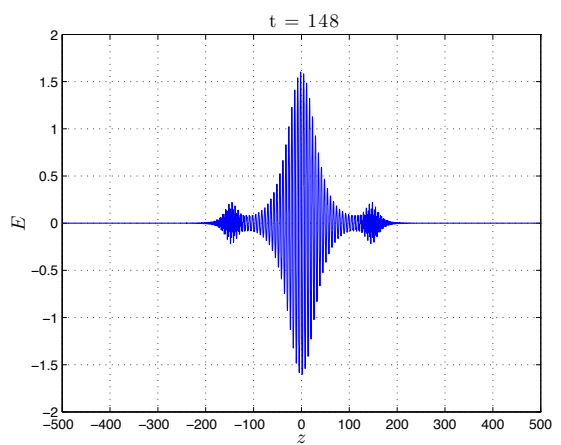

(c)

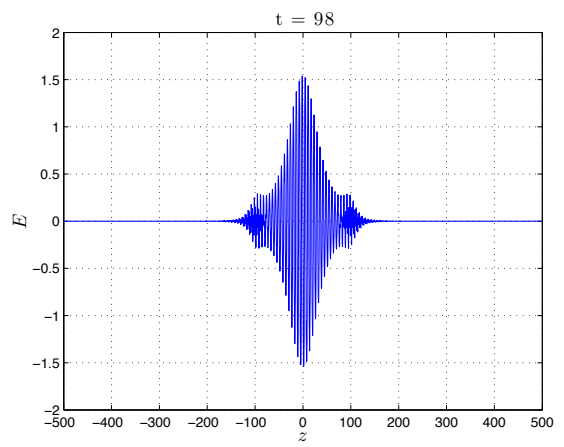

(b)

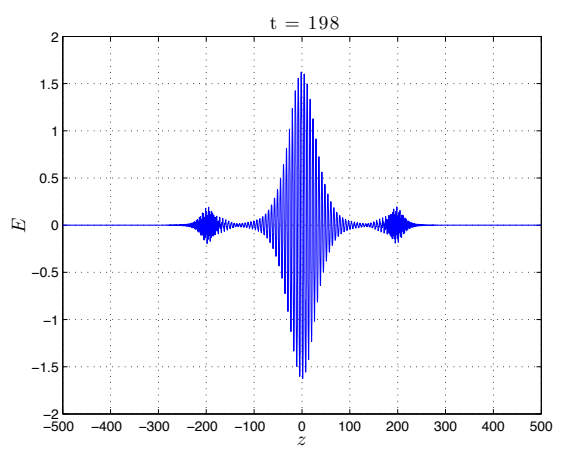

(d)

Simulations with constant refractive index, $N(z)=0$ :

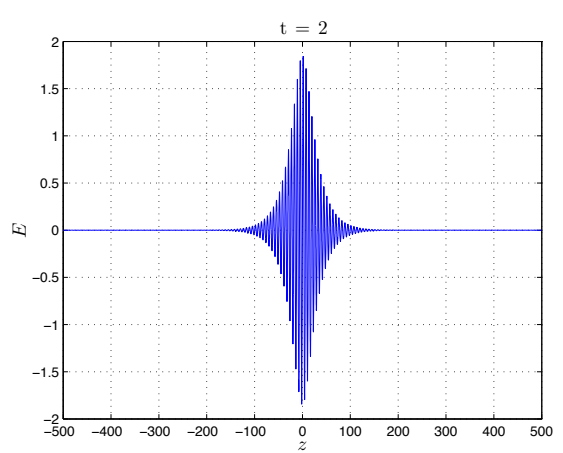

(e)

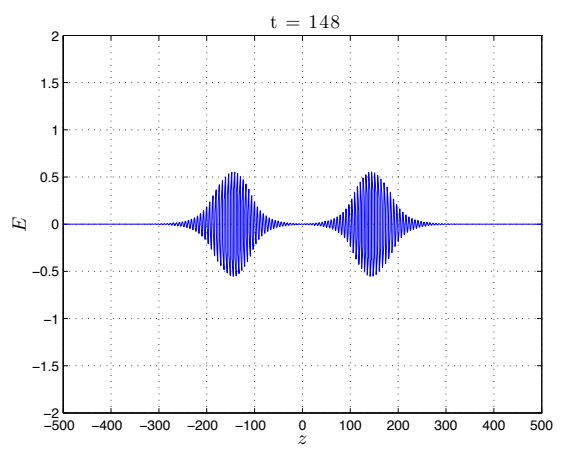

(g)

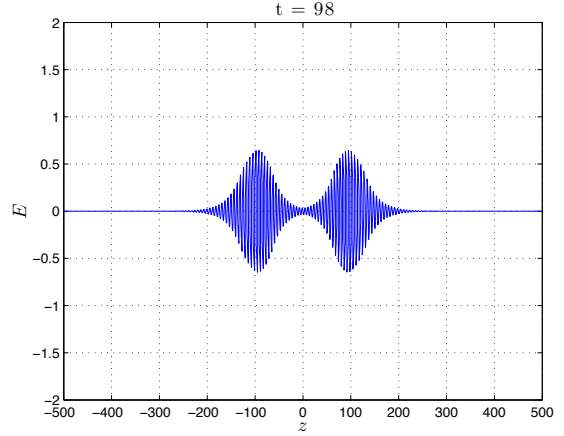

(f)

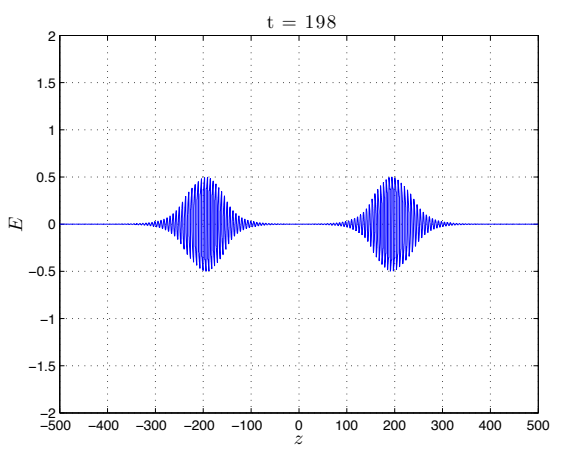

(h)

FiguRE 6. Solution of rescaled nonlinear pełtodic Maxwell equation, 3.2 , for initial data generated by the NLCME soliton with parameters $v=0$ and $\delta=\pi / 2$; see A.1. The solutions are computed with 20000 grid points on the domain $[-500,500]$. 


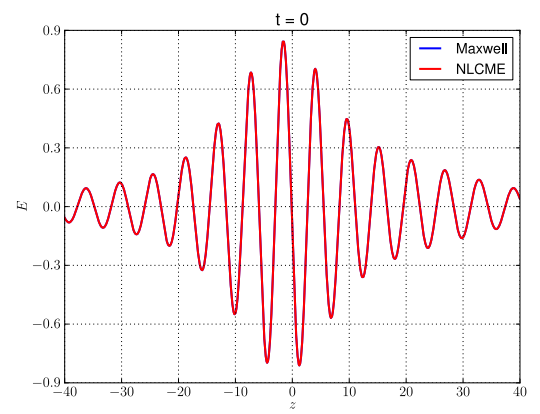

(a)

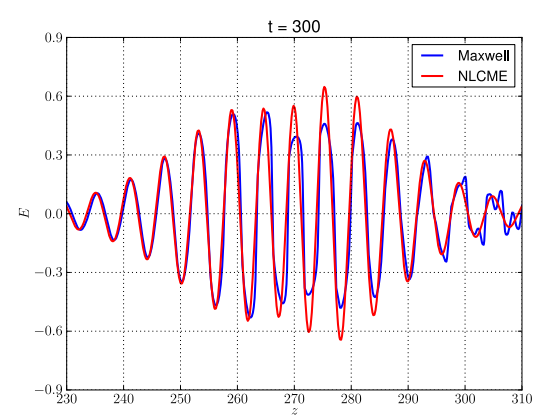

(c)

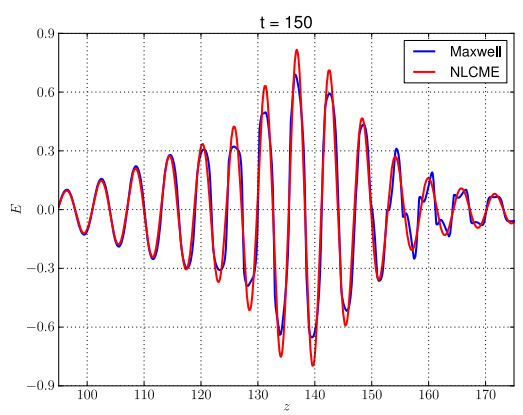

(b)

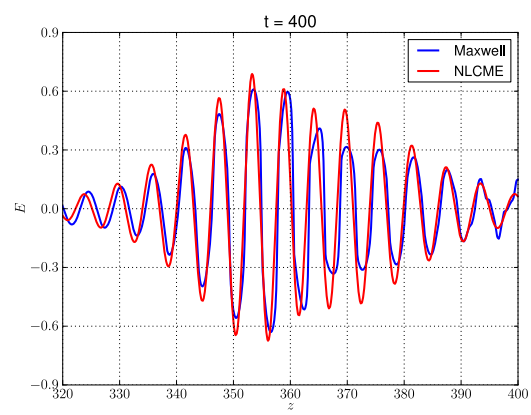

(d)

FiguRE 7. Comparison of the solution appearing in Figure $5 \mathrm{a}-\mathrm{d}$, with the exact NLCME soliton.

We note that it is also essential that the data be properly prepared to see a persistence of localization. For the initial condition

$$
\begin{aligned}
& D=0.5 \cos (z) \operatorname{sech}(\epsilon z), \\
& B=-D,
\end{aligned}
$$

we see in Figure 8 substantial spreading. This data mimics the gap soliton's amplitude, slowly varying envelope, and carrier wave, but is apparently too far outside the basin of attraction to converge to a localized state. Similar results were observed with Gaussian wave packet initial conditions.

3.2. Envelope carrier-shock trains. Although ther the slowly varying NLCME envelope shape is robust, for the nonlinear Maxwell time-evolution, there is evidence of nonlinear steepening and shock formation on the short (carrier) microstructure spatial scale. Thus, the nearly monochromatic slowly varying envelope approximation of NLCME is violated.

Figure 9 displays the time-evolution for (a) moving and (b) stationary NLCME - gap soliton data. For each initial condition, the nonlinear Maxwell evolution is simulated for different grid spacings. As we increase the number of grid points, sharp features are better resolved by the shock capturing algorithm. One can also examine the Fourier transform of the output to see that we obtain an algebraically decaying solution in wave number, with peaks at the integer wave number values.

In summary, our observations support the emergence of an envelope carrier-shock train; persistence of coherent, slowly varying, wave envelope and shock formation on the carrier scale. 


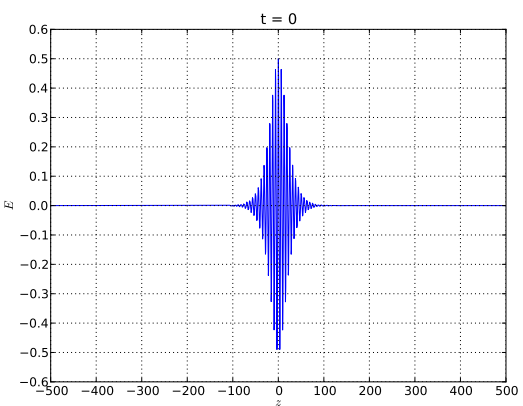

(a)

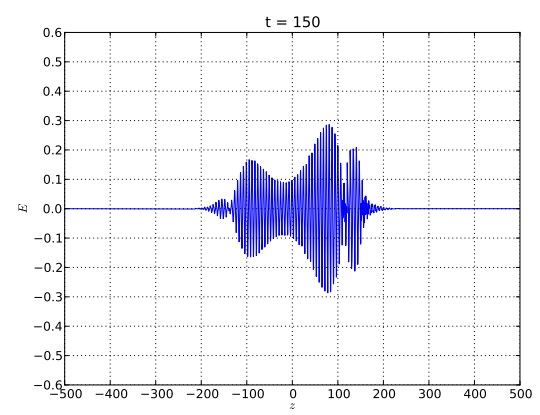

(c)

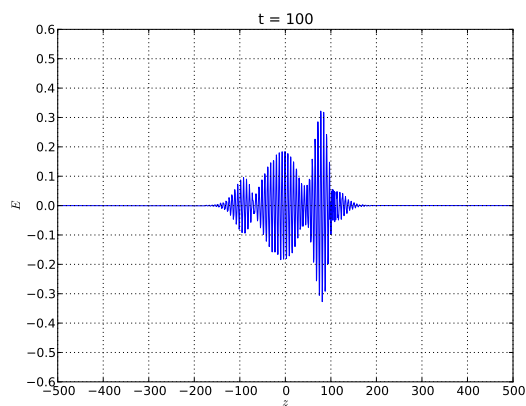

(b)

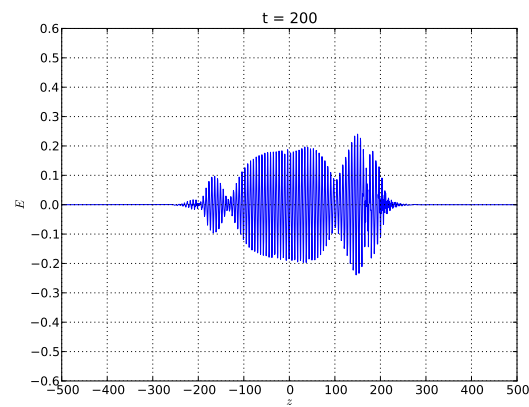

(d)

FiguRe 8. Solution of rescaled nonlinear periodic Maxwell equation, 3.2 with periodic refractive index (3.5), for initial data (3.6) . In contrast to the NLCME soliton data, the shape of the solution does not persist. The solution is computed with 20000 grid points on the domain $[-500,500]$.

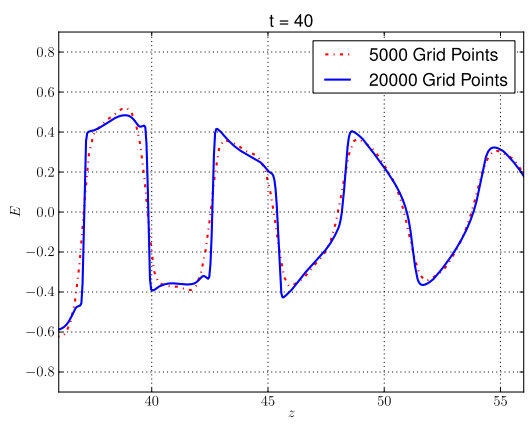

(a) $v=.9, \delta=.9$

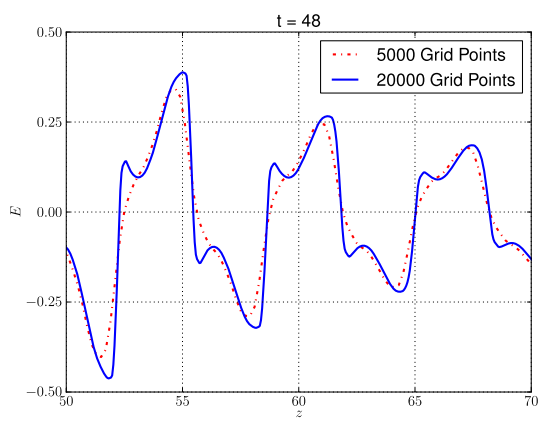

(b) $v=0, \delta=\pi / 2$

Figure 9. Increasing the number of grids points better resolves the shocks in the carrier wave. For NLCME soliton data with the indicated $v$ and $\delta$ (see (A.1)), with index of refraction $N(z)$ given by (3.5)). 


\section{Resonant NONLineAR GEOMETRICAL OPTICS AND NONLINEAR SPATially INHOMOGENEOUS MAXWELL EQUATIONS}

In this section we derive a system of equations, which incorporates all wave-resonances and which our numerical simulations show, captures the key features of the nonlinear Maxwell time-evolution, in particular, the presence of robust envelope carrier-shock train solutions. We derive this system, for general non-homogeneous media, using a nonlinear geometrical optics expansion; see, for example, [13, 14, 30]. The equations obtained are the general integro-differential equations (1.8). In the case of a periodic medium, they reduce to an infinite set of local equations, which we call the extended nonlinear coupled mode equations (xNLCME). If, in xNLCME, we neglect all but principle resonances, xNLCME reduces to NLCME.

As we shall see, in our numerical simulations of increasing high dimensional truncations of xNLCME (section 5), this theory appears to accommodate the observed carrier shocks and large scale coherent structures.

4.1. Nonlinear geometric optics expansion. In contrast to the ansatz of Section 2 , we assume the more general form

$$
\mathbf{u}(z, t)=\mathbf{u}^{(0)}(z, t, Z, T)+\epsilon \mathbf{u}^{(1)}(z, t, Z, T)+\epsilon^{2} \mathbf{u}^{(2)}(z, t, Z, T)+\ldots
$$

where $\mathbf{u}=(E, B)^{T}$ and $Z=\epsilon z, T=\epsilon t$. Inserting (4.1) into (3.2), (3.3), the first order system

$$
\partial_{t}\left(\begin{array}{c}
n(z)^{2} E+\epsilon \chi E^{3} \\
B
\end{array}\right)+\partial_{z}\left(\begin{array}{l}
-B \\
-E
\end{array}\right)=0 .
$$

we expand to get,

$$
\begin{gathered}
\left(\partial_{t}+B^{(0)} \partial_{z}\right) \mathbf{u}^{(0)}+\epsilon\left[\left(\partial_{t}+B^{(0)} \partial_{z}\right) \mathbf{u}^{(1)}+\left(\partial_{T}+B^{(0)} \partial_{Z}\right) \mathbf{u}^{(0)}\right. \\
\left.+A^{(1)}(z, \mathbf{u}) \partial_{t} \mathbf{u}^{(0)}\right]=\mathcal{O}\left(\epsilon^{2}\right)
\end{gathered}
$$

with matrices

At $\mathcal{O}\left(\epsilon^{0}\right)$,

$$
B^{(0)}=\left(\begin{array}{cc}
0 & -1 \\
-1 & 0
\end{array}\right), \quad A^{(1)}=\left(\begin{array}{cc}
2 N(z)+3 \chi E^{2} & 0 \\
0 & 0
\end{array}\right)
$$

Solving this as the generalized Eigenvalue problem,

$$
\left(B^{(0)}-\lambda I\right) \mathbf{r}=0
$$

the solutions are:

$$
\lambda_{ \pm}= \pm 1, \quad \mathbf{r}_{ \pm}=\left(\begin{array}{c}
1 \\
\mp 1
\end{array}\right)
$$

The corresponding left eigenvectors are

$$
\mathbf{l}_{ \pm}=\frac{1}{2}\left(\begin{array}{ll}
1 & \mp 1
\end{array}\right)
$$

With this normalization, $\mathbf{l}_{i} A^{(0)} \mathbf{r}_{j}=\delta_{i, j}$. The leading order fields are then

$$
\begin{aligned}
\mathbf{u}^{(0)} & =E^{+}\left(\phi_{+}, Z, T\right) \mathbf{r}_{+}+E^{-}\left(\phi_{-}, Z, T\right) \mathbf{r}_{-}, \\
E^{(0)} & =E^{+}\left(\phi_{+}, Z, T\right)+E^{-}\left(\phi_{-}, Z, T\right), \\
\phi_{ \pm} & =z \mp t .
\end{aligned}
$$

This expression is much more general than (2.6) used in the derivation of NLCME.

At $\mathcal{O}(\epsilon)$, the equation is

$$
\left(\partial_{t}+B^{(0)} \partial_{z}\right) \mathbf{u}^{(1)}=-\left(\partial_{T}+B^{(0)} \partial_{Z}\right) \mathbf{u}^{(0)}-A^{(1)}\left(z, \mathbf{u}^{(0)}\right) \partial_{t} \mathbf{u}^{(0)}
$$

If we assume

$$
\mathbf{u}^{(1)}(z, t)=m^{+}(z, t) \mathbf{r}_{+}+m^{-}(z, t) \mathbf{r}_{-},
$$


and substitute into 4.7), then left multiply by $\mathbf{l}_{+}$and then by $\mathbf{l}_{-}$, we get the two equations

$$
\begin{aligned}
-\left(\partial_{t} m^{+}+\partial_{z} m^{+}\right)= & \partial_{T} E^{+}+\partial_{Z} E^{+}+\mathbf{l}_{+} A^{(1)}\left(\mathbf{u}^{(0)}\right) \times \\
& \left(-\partial_{\phi_{+}} E^{+} \mathbf{r}_{+}+\partial_{\phi_{-}} E^{-} \mathbf{r}_{-}\right) \\
-\left(\partial_{t} m^{-}-\partial_{z} m^{-}\right)= & \partial_{T} E^{-}-\partial_{Z} E^{-}+\mathbf{l}_{-} A^{(1)}\left(\mathbf{u}^{(0)}\right) \times \\
& \left(-\partial_{\phi_{+}} E^{+} \mathbf{r}_{+}+\partial_{\phi_{-}} E^{-} \mathbf{r}_{-}\right) .
\end{aligned}
$$

The last term is the same in both equations,

$$
\begin{aligned}
\mathbf{l}_{ \pm} & A^{(1)}\left(\mathbf{u}^{(0)}\right)\left(-\partial_{\phi_{+}} E^{+} \mathbf{r}_{+}+\partial_{\phi_{-}} E^{-} \mathbf{r}_{-}\right) \\
= & \frac{1}{2}\left(2 N(z)+3 \chi E^{(0)^{2}}\right)\left(-\partial_{\phi_{+}} E^{+}+\partial_{\phi_{-}} E^{-}\right) \\
= & N(z)\left(-\partial_{\phi_{+}} E^{+}+\partial_{\phi_{-}} E^{-}\right) \\
& \quad+\frac{3}{2} \chi\left(E^{+}+E^{-}\right)^{2}\left(-\partial_{\phi_{+}} E^{+}+\partial_{\phi_{-}} E^{-}\right) .
\end{aligned}
$$

Integration of (4.9) along the characteristic $\partial_{t} z_{+}=1$ from $t=0$ to $t=L$, yields

$$
\begin{aligned}
& -\left(m^{+}\left(z_{+}(L), L\right)-m^{+}\left(z_{+}(0), 0\right)\right)= \\
& \int_{0}^{L} \partial_{T} E^{+}\left(Z, T, z_{+}(0)\right)+\partial_{Z} E^{+}\left(Z, T, z_{+}(0)\right) d s \\
& -\int_{0}^{L} N\left(z_{+}(s)\right) \partial_{\phi_{+}} E^{+}\left(Z, T, z_{+}(0)\right) d s \\
& +\int_{0}^{L} N\left(z_{+}(s)\right) \partial_{\phi_{-}} E^{-}\left(Z, T, z_{+}(s)+s\right) d s \\
& -\int_{0}^{L}\left[\frac{3}{2} \chi\left(E^{+}\left(Z, T, z_{+}(0)\right)-E^{-}\left(Z, T, z_{+}(s)+s\right)\right)^{2}\right. \\
& \left.\quad \times \partial_{\phi_{+}} E^{+}\left(Z, T, z_{+}(0)\right)\right] d s \\
& +\int_{0}^{L}\left[\frac{3}{2} \chi\left(E^{+}\left(Z, T, z_{+}(0)\right)-E^{-}\left(Z, T, z_{+}(s)+s\right)\right)^{2}\right. \\
& \left.\quad \times \partial_{\phi_{-}} E^{-}\left(Z, T, z_{+}(s)+s\right)\right] d s .
\end{aligned}
$$

Similarly, integration of 4.10 along the characteristic $\partial_{t} z_{-}=-1$, yields

$$
\begin{aligned}
&-\left(m^{-}\left(z_{-}(L), L\right)-m^{-}\left(z_{-}(0), 0\right)\right)= \\
& \int_{0}^{L} \partial_{T} E^{-}\left(Z, T, z_{+}(0)\right)-\partial_{Z} E^{-}\left(Z, T, z_{+}(0)\right) d s \\
&-\int_{0}^{L} N\left(z_{-}(s)\right) \partial_{\phi_{+}} E^{+}\left(Z, T, z_{-}(s)-s\right) d s \\
&+\int_{0}^{L} N\left(z_{-}(s)\right) \partial_{\phi_{-}} E^{-}\left(Z, T, z_{-}(0)\right) d s \\
&-\int_{0}^{L}\left[\frac{3}{2} \chi\left(E^{+}\left(Z, T, z_{-}(s)-s\right)-E^{-}\left(Z, T, z_{-}(0)\right)\right)^{2}\right. \\
&\left.\quad \times \partial_{\phi_{+}} E^{+}\left(Z, T, z_{-}(s)-s\right)\right] d s \\
&+\int_{0}^{L}\left[\frac{3}{2} \chi\left(E^{+}\left(Z, T, z_{-}(s)-s\right)-E^{-}\left(Z, T, z_{-}(0)\right)\right)^{2}\right. \\
&\left.\quad \times \partial_{\phi_{-}} E^{-}\left(Z, T, z_{-}(0)\right)\right] d s . \\
& 15
\end{aligned}
$$


Necessary conditions for $m_{ \pm}$to grow sublinearly in $t$ as $t \rightarrow \infty$ are the solvability conditions:

$$
\begin{aligned}
& \partial_{T} E^{+}\left(Z, T, z_{+}(0)\right)+\partial_{Z} E^{+}\left(Z, T, z_{+}(0)\right)= \\
& -\lim _{L \rightarrow \infty} \frac{1}{L} \int_{0}^{L} N\left(z_{+}(s)\right) \partial_{z_{+}(0)} E^{-}\left(Z, T, z_{+}(s)+s\right) d s \\
& +\lim _{L \rightarrow \infty} \frac{1}{L} \int_{0}^{L}\left[\frac{3}{2} \chi\left(E^{+}\left(Z, T, z_{+}(0)\right)+E^{-}\left(Z, T, z_{+}(s)+s\right)\right)^{2}\right. \\
& \left.\quad \times \partial_{z_{+}(0)} E^{+}\left(Z, T, z_{+}(0)\right)\right] d s, \\
& \partial_{T} E^{-}\left(Z, T, z_{-}(0)\right)-\partial_{Z} E^{-}\left(Z, T, z_{-}(0)\right)= \\
& \lim _{L \rightarrow \infty} \frac{1}{L} \int_{0}^{t} N\left(z_{-}(s)\right) \partial_{z_{-}(0)} E^{+}\left(Z, T, z_{-}(s)-s\right) d s \\
& -\lim _{L \rightarrow \infty} \frac{1}{L} \int_{0}^{L}\left[\frac{3}{2} \chi\left(E^{+}\left(Z, T, z_{-}(s)-s\right)-E^{-}\left(Z, T, z_{-}(0)\right)\right)^{2}\right. \\
& \left.\quad \partial_{z_{-}(0)} E^{-}\left(Z, T, z_{-}(0)\right)\right] d s .
\end{aligned}
$$

Given $(z, t), z_{+}(0)=z-t=\phi_{+}$and $z_{-}(0)=z+t=\phi_{-}$. Defining

$$
\langle f\rangle=\lim _{L \rightarrow \infty} \frac{1}{L} \int_{0}^{L} f(s) d s
$$

the equations may be compactly expressed as:

$$
\begin{gathered}
\partial_{T} E^{+}+\partial_{Z} E^{+}=-\left\langle N\left(\phi_{+}+s\right) \partial_{\phi} E^{-}\left(\phi_{+}+2 s\right\rangle_{s}\right. \\
+\frac{3}{2} \chi\left(\left(E^{+}\right)^{2}+2 E^{+}\left\langle E^{-}\right\rangle+\left\langle\left(E^{-}\right)^{2}\right\rangle\right) \partial_{\phi} E^{+} \\
\partial_{T} E^{-}-\partial_{Z} E^{-}=\left\langle N\left(\phi_{-}-s\right) \partial_{\phi} E^{+}\left(\phi_{-}-2 s\right)\right\rangle_{s} \\
-\frac{3}{2} \chi\left(\left(E^{-}\right)^{2}+2 E^{-}\left\langle E^{+}\right\rangle+\left\langle\left(E^{+}\right)^{2}\right\rangle\right) \partial_{\phi} E^{-} .
\end{gathered}
$$

It is important to recognize that the arguments of the fields in $4.16 \mathrm{a}$ are $\phi_{+}=z-t, Z$, and $T$, while in $4.16 \mathrm{~b}$, they are $\phi_{-}=z+t, Z$, and $T$. As in our derivation of NLCME in Section $2, \Gamma \equiv \frac{3}{2} \chi$. With this notation, 4.16 can be rewritten, after an integration by parts, in conservation law form,

$$
\begin{gathered}
\partial_{T} E^{+}+\partial_{Z} E^{+}=\partial_{\phi}\left\langle n_{1}\left(\phi_{+}+s\right) E^{-}\left(\phi_{+}+2 s\right\rangle_{s}\right. \\
+\Gamma \partial_{\phi}\left[\frac{1}{3}\left(E^{+}\right)^{3}+\left(E^{+}\right)^{2}\left\langle E^{-}\right\rangle+E^{+}\left\langle\left(E^{-}\right)^{2}\right\rangle\right], \\
\partial_{T} E^{-}-\partial_{Z} E^{-}=-\partial_{\phi}\left\langle n_{1}\left(\phi_{-}-s\right) E^{+}\left(\phi_{-}-2 s\right)\right\rangle_{s} \\
-\Gamma \partial_{\phi}\left[\left\langle\left(E^{+}\right)^{2}\right\rangle E^{-}+\left\langle E^{+}\right\rangle\left(E^{-}\right)^{2}+\frac{1}{3}\left(E^{-}\right)^{3}\right] .
\end{gathered}
$$

Equations 4.17 corresponds to the integro-differential equations of the introduction, if we omit the $\left\langle E^{ \pm}\right\rangle$ terms. Since $\left\langle E^{ \pm}\right\rangle$is time-invariant (see section 4.3 by choosing initial conditions for which $\left\langle E^{ \pm}\right\rangle(T=0)=$ 0 , these terms can be dropped from (4.17). Finally, note that 4.16 are applicable to a general heterogeneous dielectric material with the appropriate scalings.

4.2. Periodic Media and xNLCME. We now specialize to the periodic case. Assume now that $N(z+$ $2 \pi)=N(z)$. Then (4.17) is invariant under the discrete translation: $\phi \mapsto \phi+2 \pi$, i.e.

$$
\begin{aligned}
& E^{+}(\phi, Z, T) \mapsto E^{+}(\phi+2 \pi, Z, T) \\
& E^{-}(\phi, Z, T) \mapsto E^{-}(\phi+2 \pi, Z, T) .
\end{aligned}
$$

Thus, under the assumption of existence and uniqueness of solutions to (4.17), if the initial data are $2 \pi$ in the $\phi$ argument, then the solutions remain $2 \pi$ periodic in $\phi$. In the periodic setting, the averaging operator, 
4.15, simplifies to

$$
\langle f\rangle=\frac{1}{2 \pi} \int_{0}^{2 \pi} f(s) d s .
$$

We now expand $N(z)$ and $E^{ \pm}$in Fourier series,

$$
\begin{aligned}
N(z) & =\sum_{p \in \mathbb{Z}} N_{p} e^{\mathrm{i} p z}, \\
E^{ \pm}(\phi, Z, T) & =\sum_{p} E_{p}^{ \pm}(Z, T) e^{ \pm \mathrm{i} p \phi},
\end{aligned}
$$

where $\bar{N}_{p}=N_{-p}$ and $\bar{E}_{p}^{ \pm}=E_{-p}^{ \pm}$since $N$ and $E^{ \pm}$are real valued. In this case, the system of Fourier coefficients $\left\{E_{p}^{ \pm}(Z, T): p \in \mathbb{Z}\right\}$ satisfy the infinite system of extended nonlinear coupled mode equations (xNLCME):

$$
\begin{aligned}
\partial_{T} E_{p}^{+}+\partial_{Z} E_{p}^{+}= & \mathrm{i} p N_{2 p} E_{p}^{-}+\mathrm{i} p \frac{\Gamma}{3}\left[\sum_{q, r} E_{q}^{+} E_{r}^{+} E_{p-q-r}^{+}\right. \\
& \left.+3 E_{0}^{-} \sum_{q} E_{q}^{+} E_{p-q}^{+}+3\left(\sum_{q}\left|E_{q}^{-}\right|^{2}\right) E_{p}^{+}\right], \\
\partial_{T} E_{p}^{-}-\partial_{Z} E_{p}^{-}= & \mathrm{i} p \bar{N}_{2 p} E_{p}^{+}+\mathrm{i} p \frac{\Gamma}{3}\left[\sum_{q, r} E_{q}^{-} E_{r}^{-} E_{p-q-r}^{-}\right. \\
& \left.+3 E_{0}^{+} \sum_{q} E_{q}^{-} E_{p-q}^{-}+3\left(\sum_{q}\left|E_{q}^{+}\right|^{2}\right) E_{p}^{-}\right] .
\end{aligned}
$$

4.3. Conservation Laws and Hamiltonian Structure. Equation (4.17), and alternatively (4.21), have two conservation laws:

Proposition 4.1. Assume that $E^{ \pm}$is a sufficiently smooth and sufficiently $Z$ - decaying solution of 4.17) and that $\left\{E_{p}(Z, T)\right\}_{p \in \mathbb{Z}}$ is the corresponding solution of $x N L C M E$. Then,

$$
\begin{gathered}
\frac{d}{d T} \int\left\langle E^{+}(\cdot, T)\right\rangle d Z=\frac{d}{d T} \int E_{0}^{+}(\cdot, T) d Z=0 \\
\frac{d}{d T} \int\left\langle E^{+}(\cdot, T)\right\rangle d Z=\frac{d}{d T} \int E_{0}^{-}(\cdot, T) d Z=0 \\
\frac{d}{d T} \int\left\langle\left(E^{+}\right)^{2}(\cdot, T)\right\rangle+\left\langle\left(E^{-}\right)^{2}(\cdot, T)\right\rangle d Z \\
=\frac{d}{d T} \sum_{p} \int\left|E_{p}^{+}(\cdot, T)\right|^{2}+\left|E_{p}^{-}(\cdot, T)\right|^{2} d Z=0 .
\end{gathered}
$$

Proof. Setting $p=0$ in 4.21,

$$
\begin{aligned}
& \partial_{T} E_{0}^{+}+\partial_{Z} E_{0}^{+}=0, \\
& \partial_{T} E_{0}^{-}-\partial_{Z} E_{0}^{-}=0 .
\end{aligned}
$$

Integrating in $Z$ establishes the first two conservation laws in terms of the Fourier modes. Integrating 4.20 in $\phi$ over $[0,2 \pi)$ relates $\left\langle E^{ \pm}\right\rangle$to $E_{0}^{ \pm}$. 
Multiplying 4.21a by $\bar{E}_{p}^{+}$, summing over $p$, and adding its complex conjugate,

$$
\begin{aligned}
\sum \partial_{T}\left|E_{p}^{+}\right|^{2}+\partial_{Z}\left|E_{p}^{+}\right|^{2}= & \sum_{p} i p N_{2 p} E_{p}^{-} \bar{E}_{p}^{+}+\frac{\Gamma}{3} \sum_{p} i p\left[\sum_{q, r} E_{q}^{+} E_{r}^{+} E_{-p}^{+} E_{p-q-r}^{+}\right. \\
& +3 E_{0}^{-} \sum_{q} E_{q}^{+} E_{p-q}^{+} E_{-p}^{+} \\
& \left.+3\left(\sum_{q}\left|E_{q}^{-}\right|^{2}\right)\left|E_{p}^{+}\right|^{2}\right]+ \text { c.c. }
\end{aligned}
$$

The quartic terms will all vanish. Consider the first quartic term, and note that

$$
\begin{aligned}
\sum_{p, q, r} p E_{q}^{+} E_{r}^{+} E_{-p}^{+} E_{p-q-r}^{+} & =\sum_{k_{1}+k_{2}+k_{3}+k_{4}=0} k_{1} E_{k_{1}}^{+} E_{k_{2}}^{+} E_{k_{3}}^{+} E_{k_{4}}^{+} \\
& =\sum_{k_{1}+k_{2}+k_{3}+k_{4}=0} k_{2} E_{k_{1}}^{+} E_{k_{2}}^{+} E_{k_{3}}^{+} E_{k_{4}}^{+} \\
& =\sum_{k_{1}+k_{2}+k_{3}+k_{4}=0} k_{3} E_{k_{1}}^{+} E_{k_{2}}^{+} E_{k_{3}}^{+} E_{k_{4}}^{+} \\
& =\sum_{k_{1}+k_{2}+k_{3}+k_{4}=0} k_{4} E_{k_{1}}^{+} E_{k_{2}}^{+} E_{k_{3}}^{+} E_{k_{4}}^{+}
\end{aligned}
$$

Hence,

$$
\begin{aligned}
& \sum_{p, q, r} p E_{q}^{+} E_{r}^{+} E_{-p}^{+} E_{p-q-r}^{+} \\
& \quad=\frac{1}{4} \sum_{k_{1}+k_{2}+k_{3}+k_{4}=0}\left(k_{1}+k_{2}+k_{3}+k_{4}\right) E_{k_{1}}^{+} E_{k_{2}}^{+} E_{k_{3}}^{+} E_{k_{4}}^{+}=0
\end{aligned}
$$

The second quartic term vanishes using a similar analysis. The last quartic term,

$$
\sum_{p} p\left(\sum_{q}\left|E_{q}^{-}\right|^{2}\right)\left|E_{p}^{+}\right|^{2}
$$

will vanish because the $p$ and $-p$ terms will cancel one another. Similar analysis holds for 4.21b), leaving us with the two equations

$$
\begin{aligned}
& \sum \partial_{T}\left|E_{p}^{+}\right|^{2}+\partial_{Z}\left|E_{p}^{+}\right|^{2}=\sum i p N_{2 p} E_{p}^{-} \bar{E}_{p}^{+}-i p \bar{N}_{2 p} \bar{E}_{p}^{-} E_{p}^{+}, \\
& \sum \partial_{T}\left|E_{p}^{-}\right|^{2}-\partial_{Z}\left|E_{p}^{-}\right|^{2}=\sum i p \bar{N}_{2 p} \bar{E}_{p}^{-} E_{p}^{+}-i p N_{2 p} E_{p}^{-} \bar{E}_{p}^{+} .
\end{aligned}
$$

Summing these two, and integrating in $Z$ gives the $L^{2}$ conservation law.

To simplify our analysis we assume $E_{0}^{ \pm}$are initially zero from here on. The equations reduce to

$$
\begin{aligned}
\partial_{T} E^{+}+\partial_{Z} E^{+}= & \partial_{\phi}\left\langle N\left(\phi_{+}+s\right) E^{-}\left(\phi_{+}+2 s\right\rangle_{s}\right. \\
& +\Gamma \partial_{\phi}\left[\frac{1}{3}\left(E^{+}\right)^{3}+E^{+}\left\langle\left(E^{-}\right)^{2}\right\rangle\right], \\
\partial_{T} E^{-}-\partial_{Z} E^{-}= & -\partial_{\phi}\left\langle N\left(\phi_{-}-s\right) E^{+}\left(\phi_{-}-2 s\right)\right\rangle_{s} \\
& -\Gamma \partial_{\phi}\left[\left\langle\left(E^{+}\right)^{2}\right\rangle E^{-}+\frac{1}{3}\left(E^{-}\right)^{3}\right] .
\end{aligned}
$$


and

$$
\begin{aligned}
& \partial_{T} E_{p}^{+}+\partial_{Z} E_{p}^{+}=\mathrm{i} p N_{2 p} E_{p}^{-}+\mathrm{i} p \frac{\Gamma}{3}[ \sum E_{q}^{+} E_{r}^{+} E_{p-q-r}^{+} \\
&\left.+3\left(\sum\left|E_{q}^{-}\right|^{2}\right) E_{p}^{+}\right], \\
& \partial_{T} E_{p}^{-}-\partial_{Z} E_{p}^{-}=\mathrm{i} p \bar{N}_{2 p} E_{p}^{+}+\mathrm{i} p \frac{\Gamma}{3}[ \sum E_{q}^{-} E_{r}^{-} E_{p-q-r}^{-} \\
&\left.+3\left(\sum\left|E_{q}^{+}\right|^{2}\right) E_{p}^{-}\right] .
\end{aligned}
$$

These are equations 1.8 and 1.10 from the introduction. Truncating 4.29 to just mode $E_{ \pm 1}^{ \pm}$, recovers the NLCME, subject to the identification of $\mathcal{E}^{ \pm}$with $E_{1}^{ \pm}$.

Another time-invariant functional is a consequence of the Hamiltonian structure given in the following result, which is straightforward to verify:

Proposition 4.2. The system 4.29 is a Hamiltonian system:

$$
\partial_{T} E_{p}^{+}=-\mathrm{i} p \frac{\delta H}{\delta \bar{E}_{p}^{+}}, \quad \partial_{T} E_{p}^{-}=-\mathrm{i} p \frac{\delta H}{\delta \bar{E}_{p}^{-}},
$$

where with time-invariant Hamiltonian

$$
H\left[E^{ \pm}, \overline{E^{ \pm}}\right]=\int \mathcal{H}(\cdot, T)
$$

and Hamiltonian density

$$
\begin{aligned}
\mathcal{H}(Z, T)= & \frac{\mathrm{i}}{2} \sum_{p_{1}=1}^{\infty} \frac{1}{p_{1}}\left(E_{p_{1}}^{+} \partial_{Z} \bar{E}_{p_{1}}^{+}-E_{p_{1}}^{-} \partial_{Z} \bar{E}_{p_{1}}^{-}\right)-\sum_{p_{1}=1}^{\infty} N_{2 p_{1}} \bar{E}_{p_{1}}^{+} E_{p_{1}}^{-} \\
& -\frac{\Gamma}{3} \frac{1}{2} \frac{1}{4} \sum_{p_{1}+p_{2}+p_{3}+p_{4}=0} E_{p_{1}}^{+} E_{p_{2}}^{+} E_{p_{3}}^{+} E_{p_{4}}^{+}+E_{p_{1}}^{-} E_{p_{2}}^{-} E_{p_{3}}^{-} E_{p_{4}}^{-} \\
& -\Gamma \frac{1}{2} \frac{1}{2}\left(\sum_{p_{1}}\left|E_{p_{1}}^{+}\right|^{2}\right)\left(\sum_{p_{1}}\left|E_{p_{1}}^{-}\right|^{2}\right)+\text { c.c. } .
\end{aligned}
$$

\section{Simulations of the Truncated xNLCME}

In this section we simulate truncations of the infinite dimensional xNLCME system, performed pseudospectrally with fourth order Runge-Kutta time stepping. These simulations suggest that

- xNLCME has its own localized soliton-like structures which better capture the dynamics of the nonlinear periodic Maxwell equation for our class of initial conditions than NLCME and

- xNLCME has singular solutions, $\left\{E_{p}^{ \pm}(Z, T)\right\}$ with a cascade of energy to higher wave numbers, $p$. The physical electric field

$$
\begin{aligned}
E(z, t) \approx \epsilon^{\frac{1}{2}}\left(E^{+}(z-t,, \epsilon z, \epsilon t)+E^{-}(z+t,, \epsilon z, \epsilon t)\right) \\
=\epsilon^{\frac{1}{2}} \sum_{p \in \mathbb{Z} \backslash 0}\left(E_{p}^{+}(Z, T) e^{i p(Z-T) / \epsilon}+E_{p}^{-}(Z, T) e^{-i p(Z+T) / \epsilon}\right) \\
\quad+\text { c.c. }
\end{aligned}
$$

develops a carrier-shock train structure.

As we saw in Section 3.1. particularly Figure 6, though the NLCME soliton data appeared robust, there was some escape of energy. This can be accounted for in the xNLCME through the inclusion of additional modes.

Starting with the same initial conditions, we simulate the NLCME soliton of $E_{ \pm 1}^{ \pm}$with soliton parameters $v=0$ and $\delta=\frac{\pi}{2}$, and material parameters

$$
\Gamma=1, \quad N_{ \pm 2}=\frac{2}{\pi}, \quad N_{j \neq \pm 2}=0
$$



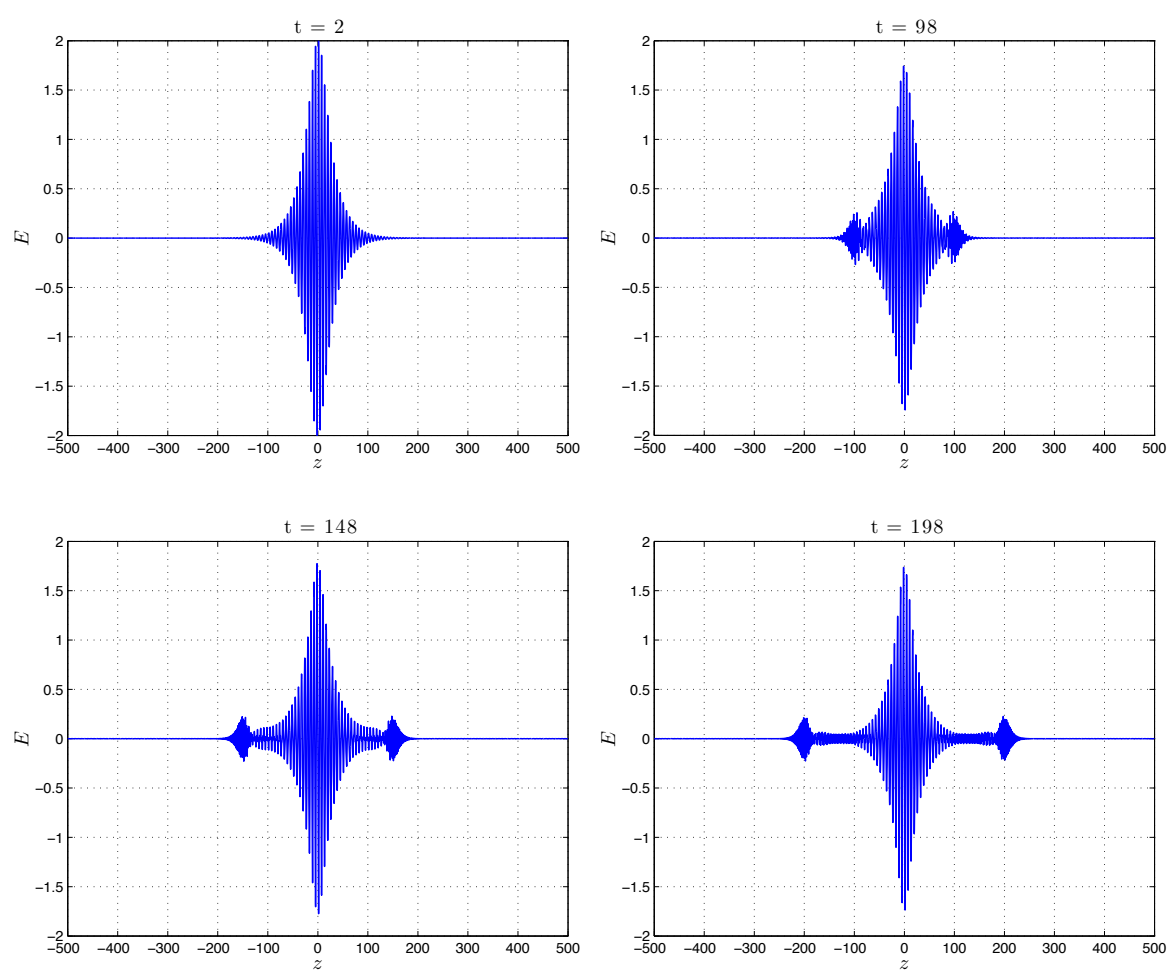

Figure 10. Evolution of an NLCME soliton in the xNLCME, resolving odd modes $|p| \leq 4$. Computed with 4096 grid points in the $Z$ coordinate. Compare with Figure 6 .

in 4.29 resolving only a finite number of harmonics. The primitive electric field is reconstructed from these simulations as

$$
E=\sum_{p=-p_{\max }}^{p_{\max }} E_{p}^{+}(Z, T) e^{i p(Z-T) / \epsilon}+E_{p}^{-}(Z, T) e^{-i p(Z+T) / \epsilon}+\text { c.c. }
$$

$E$ is plotted in Figures 10, and 11, which resolve odd modes up to 3 and 15, respectively. Comparing with Figure 6, we infer that the two smaller pulses symmetrically expelled from the main wave were transferred into $E_{ \pm 3}^{ \pm}$, since these clearly appear in Figure 10 . This addresses the macroscopic discrepancy between NLCME and Maxwell.

Including the additional modes also suggests shock formation by re-examining Figure 9. The sharper, shock like features, can only be resolved by the inclusion of the the higher harmonics. The contrast between different truncations is shown in Figure 12. Indeed, we see the Gibbs phenomenon that would be expected from taking a truncated Fourier representation of a discontinuous function.

Despite this, NLCME still gets certain leading order effects correct, such as the main structure in the Maxwell simulations. The robustness of NLCME can also be seen by exploring how energy is partitioned amongst the harmonics. Let

$$
e_{p} \equiv \int\left(\left|E_{p}^{+}\right|^{2}+\left|E_{-p}^{+}\right|^{2}+\left|E_{p}^{-}\right|^{2}+\left|E_{-p}^{-}\right|^{2}\right) d Z, \quad p=1,3, \ldots p_{\max }
$$

This is the energy associated with mode $p$. Their sum is conserved. Plotting this for the above simulations in Figure 13, we see that most of the energy remains in mode one, some migrates into mode three, and less in the subsequent modes. 

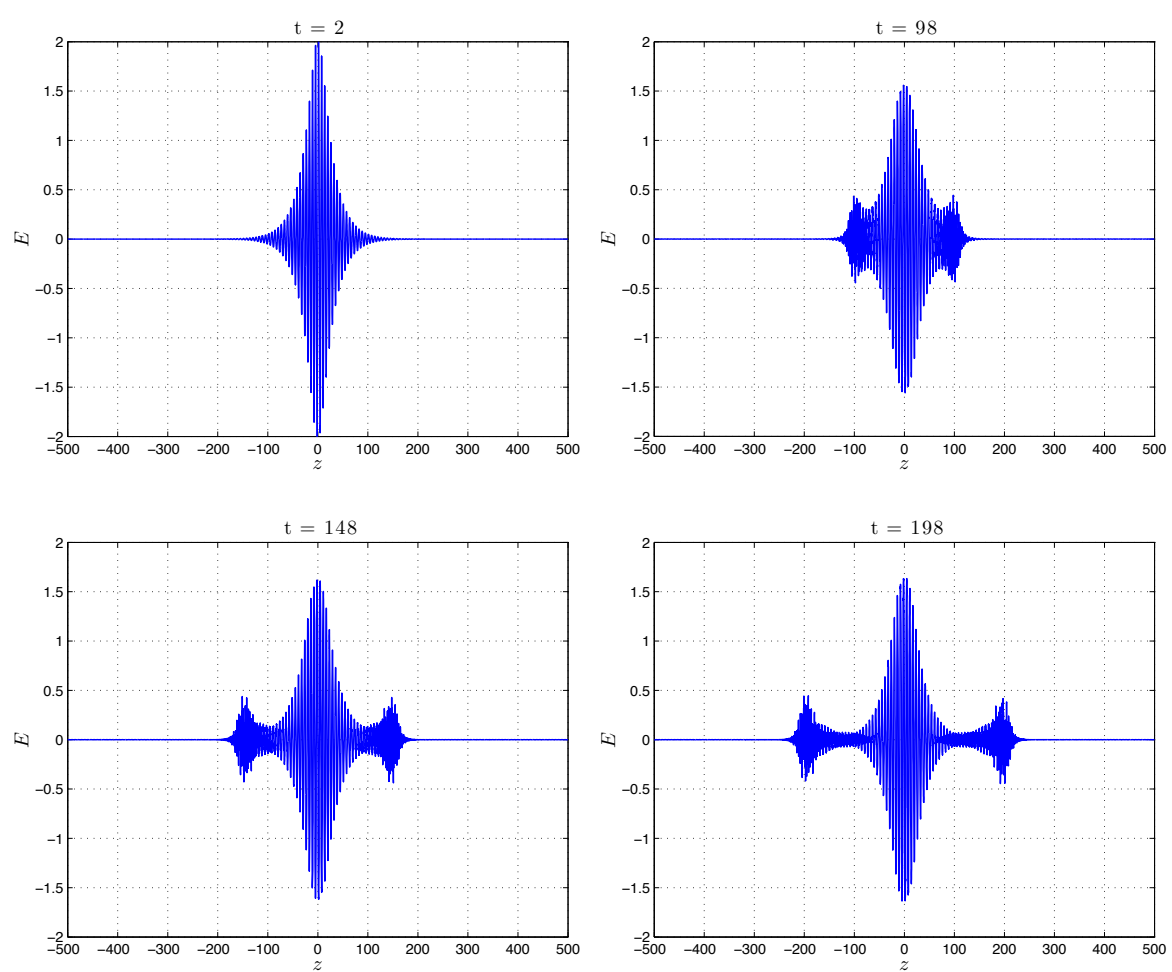

FigURE 11. Evolution of an NLCME solition in the xNLCME, resolving odd modes $|p| \leq 16$. Computed with 16384 grid points in the $Z$ coordinate. Compare with Figure 6 .

\section{Summary AND DISCUSSION}

We first numerically simulated the one-dimensional nonlinear Maxwell equations in the regime of weak nonlinearity, low contrast periodic structure (weak dispersion) with wave-packet data satisfying a Bragg resonance condition, i.e. carrier wavelength equal to twice the medium periodicity. We observe strong evidence of the emergence of a coherent structure evolving as slowly varying envelope with a carrier-shock train. This violates the nearly-monochromatic assumption underlying the classical nonlinear coupled mode equations. We propose our nonlocal integro-differential equations governing coupled forward and backward waves, derived via a nonlinear geometrical optics expansion, as the physically correct, mathematically consistent description of waves governed by nonlinear Maxwell in a periodic structure with negligible chromatic (nonlocal in time) dispersion. These equations are equivalent to an infinite dimensional system of couple first order PDEs, the extended coupled mode system (xNLCME). The electric field, E, obtained from numerical solution of successively higher truncations of xNLCME converges toward the envelope carrier-shock trains observed in direct simulations of the nonlinear Maxwell equations.

Finally we mention that our methods could be applied to study the long time evolution of wave-packet type initial conditions for the problem of quadratically nonlinear elastic media, consider in $17,25,26$ We obtain nonlocal equations of resonant nonlinear geometrical optics (or equivalently an infinite family of nonlinear coupled mode equations), governing interacting forward and backward propagating waves 42. A difference between the quadratic and cubic case is that the smallest truncated system that retains nonlinear interactions contains four modes, $p= \pm 1, \pm 2$. Nonlinear effects occur through second harmonic generation, a process well-known in nonlinear optics.

Open problems and conjectures: As our simulations show, there is agreement between finite mode truncations of the integro-differential equations and the primitive Maxwell system. Assessing, and proving the time of validity of this approximation is one upon problem. 


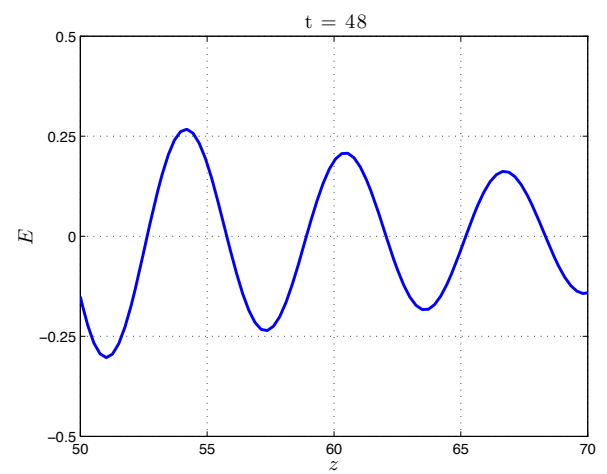

(a) Resolves odd harmonics $|p| \leq 2$

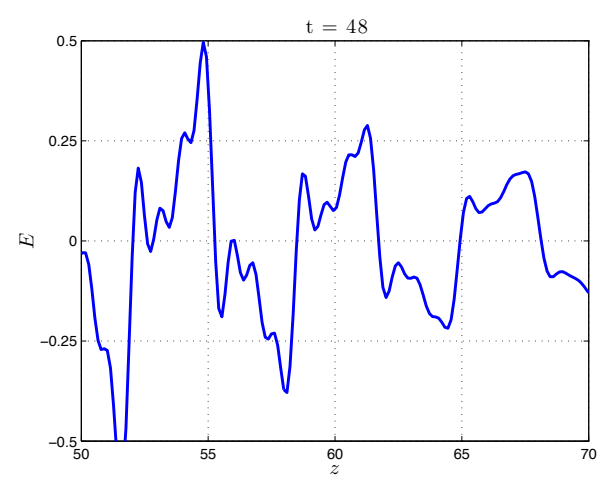

(c) Resolves odd harmonics $|p| \leq 8$

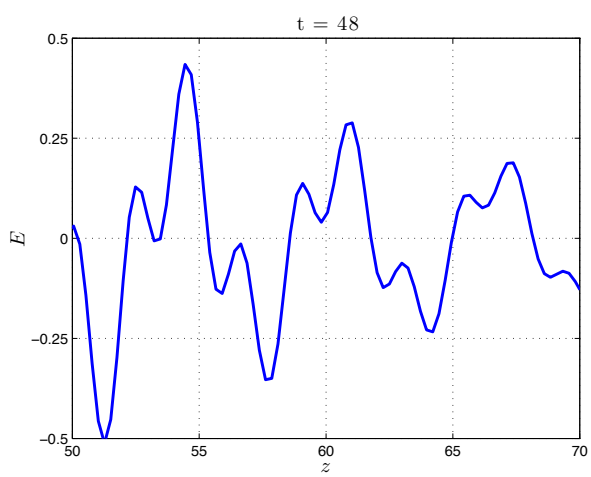

(b) Resolves odd harmonics $|p| \leq 4$

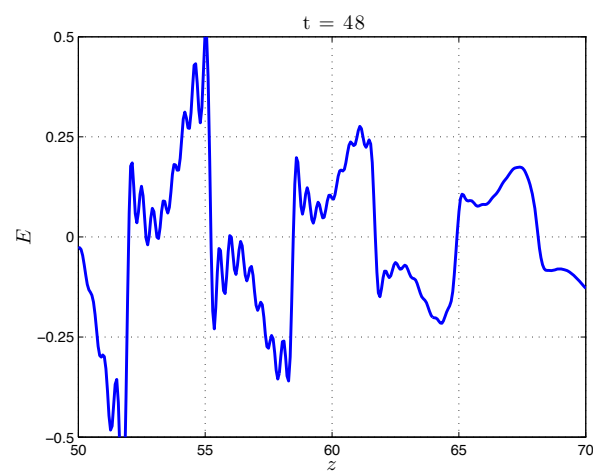

(d) Resolves odd harmonics $|p| \leq 16$

Figure 12. Comparison of the features that develop on the scale of the medium in different truncations of the equations. Including additional harmonics better captures the shocks seen in Figure 9

Following up on assessing the time of validity, there is also the question of the time of existence and the well-posedness of the equations. We expect that solutions of xNLCME for initial data having a finite number of nonzero mode amplitudes, e.g. NLCME gap soliton data, will give rise to solutions of xNLCME that develop singularities in finite time. The nature of this blowup is expected to occur via a cascade to high mode amplitudes (higher index, $p$ ), corresponding to modes necessary to resolve the carrier shock structure in the small scale. As we mentioned in the discussion, there is clearly singularity formation when the heterogeneity is turned off $(N=0)$, and either $E^{+}$or $E^{-}$is initially zero. It is an open problem as to whether this particular mechanism for singularity formation will persist when coupling is restored.

As pointed out in the introduction, the success in modeling experiments with NLCME suggests that, although there is such a (weakly turbulent) cascade, it is only a small part of the optical power that is transferred to high wavenumbers and that this energy contributes mainly to resolving the small-scale shocks. To explore this, one needs to simulate the xNLCME equations with many more harmonics. Plotting the Fourier transform (in the $Z$ coordinate) of the simulations in Section 5 in figure 14 , we see that the spectral support grows as we increase the number of resolved envelopes (the $E_{p}^{ \pm}$'s). A related question is whether or not the primitive Maxwell system, the xNLCME system, or one of its truncations possess genuine coherent structures. In [46], the authors found such solutions for a first and third harmonic system. This shall be further explored in the forthcoming publication, 36 .

Finally, our computations in Section 3 invoked of a gas-dynamics entropy condition. Such a condition is necessary to use finite volume methods. Although thermodynamically consistent, we do not know whether this is the correct regularizing mechanism of electrodynamics. 


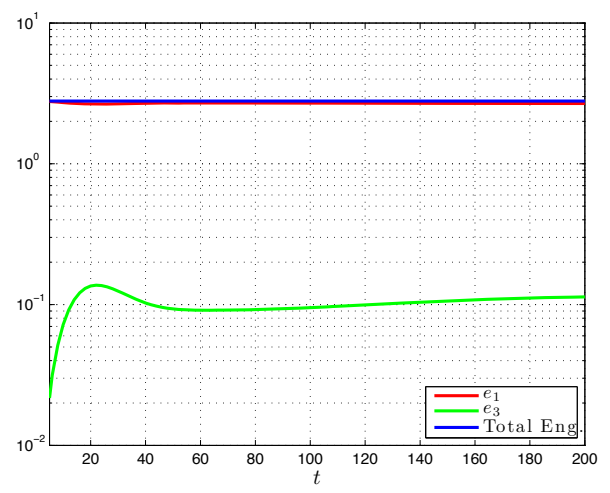

(a) Resolves odd harmonics $|p| \leq 4$

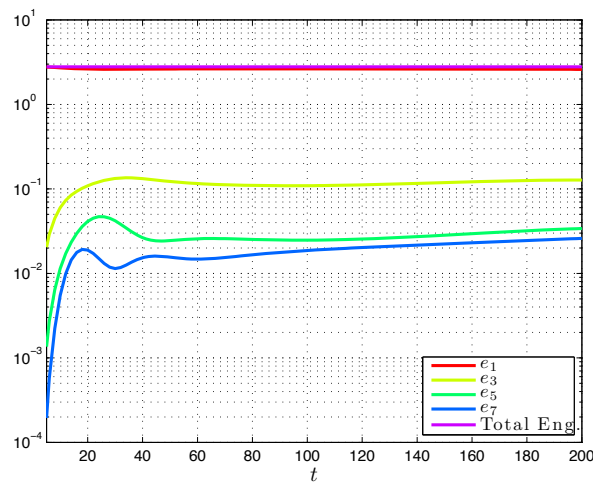

(b) Resolves odd harmonics $|p| \leq 8$

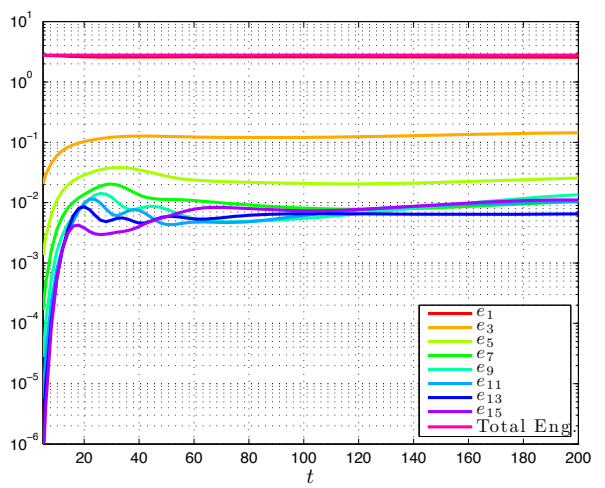

(c) Resolves odd harmonics $|p| \leq 16$

FiguRE 13. Energy distribution, (5.2), for truncated xNLCME simulations with different numbers of harmonics. In all cases, the energy initially residing in mode one tends to stay there.

\section{REFERENCES}

[1] A. Aceves and S. Wabnitz. Self-induced transparency solitons in nonlinear refractive periodic media. Physics Letters A, 141(1-2), 1989.

[2] D. Bale, R. LeVeque, S. Mitran, and J. Rossmanith. A wave propagation method for conservation laws and balance laws with spatially varying flux functions. SIAM Journal on Scientific Computing, 24(3):955-978, 2003.

[3] D. Christodoulides and Joseph. Slow Bragg solitons in nonlinear periodic structures. Phys. Rev. Lett., 62(15):1746-1749, 1989.

[4] C. de Sterke and J. Sipe. Gap solitons. Progress in Optics, 33:205-205, 1994.

[5] F. DeMartini, C. Townes, T. Gustafson, and P. Kelley. Self-steepening of light pulses. Physical Review, 164(2):312-323, Jan 1967.

[6] P. Donnat and J. Rauch. Dispersive nonlinear geometric optics. Journal of Mathematical Physics, 38(3):1484-1523, Mar 1997.

[7] M. Eastham. The Spectral Theory of Periodic Differential Equations. Scottish Academic Press, 1973.

[8] B. J. Eggleton, C. M. de Sterke, and R. E. Slusher. Nonlinear pulse propagation in Bragg gratings. J. . Opt. Soc. Am. B, 14:2980-2992, 1997.

[9] R. Flesch, A. Pushkarev, and J. Moloney. Carrier wave shocking of femtosecond optical pulses. Physical review letters, 76(14):2488-2491, 1996.

[10] R. Goodman, R. Slusher, and M. Weinstein. Stopping light on a defect. J Opt Soc Am B, 19(7):1635-1652, 2002.

[11] R. H. Goodman, M. I. Weinstein, and P. Holmes. Nonlinear propagation of light in one-dimensional periodic structures. Journal of Nonlinear Science, 11:123-168, 2001.

[12] M. Groves and G. Schneider. Modulating pulse solutions for a class of nonlinear wave equations. Communications in Mathematical Physics, 219(3):489-522, 2001.

[13] J. Hunter and J. Keller. Weakly nonlinear high frequency waves. Communications on Pure and Applied Mathematics, 36:547-569, 1983. 


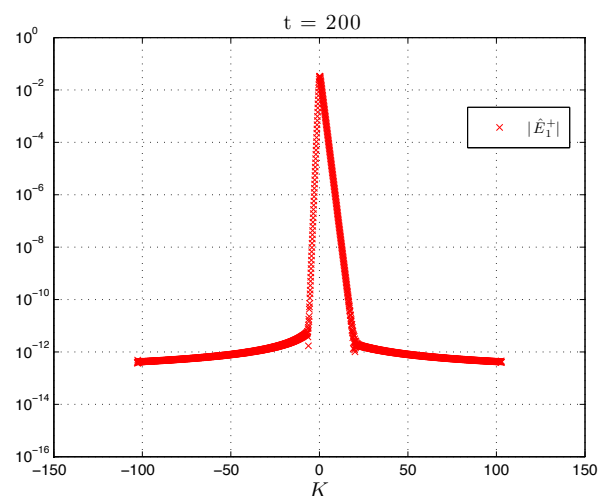

(a) Resolves odd harmonics $|p| \leq 2$

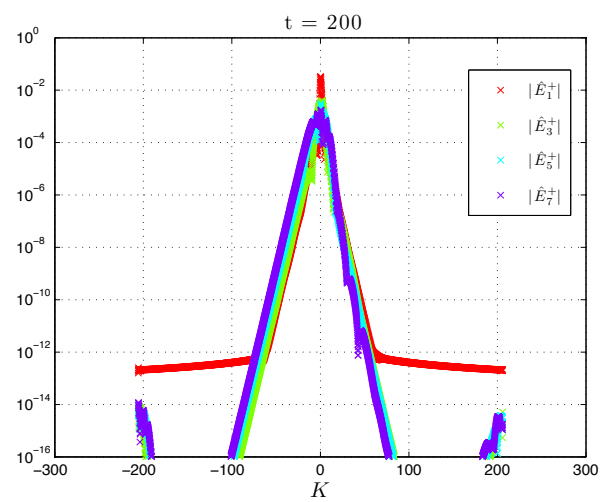

(c) Resolves odd harmonics $|p| \leq 8$

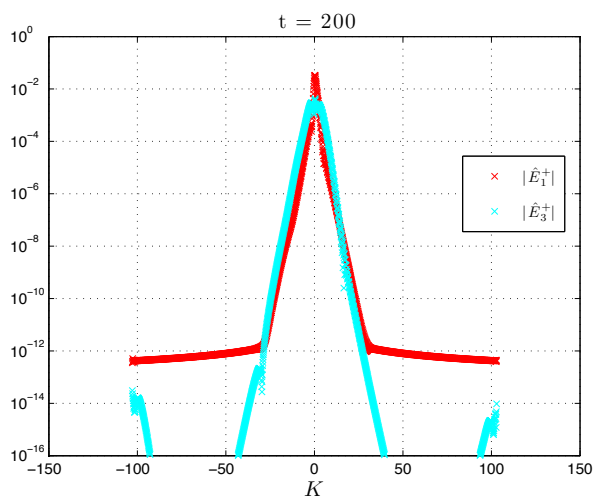

(b) Resolves odd harmonics $|p| \leq 4$

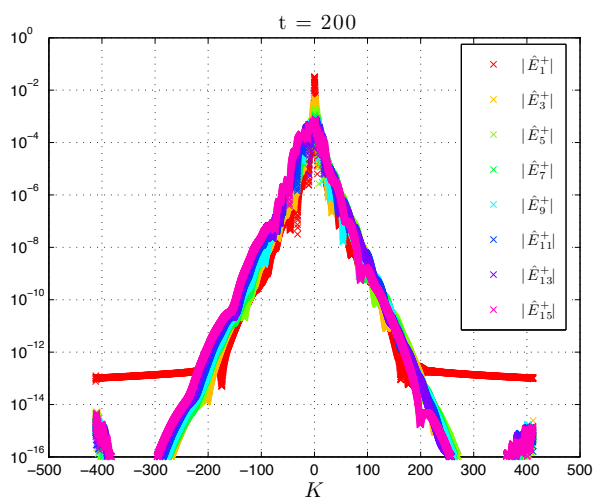

(d) Resolves odd harmonics $|p| \leq 16$

Figure 14. Fourier transforms of the solutions to truncations of the xNLCME equations. Increasing the number of envelopes expands the support in Fourier space.

[14] J. Hunter, A. Majda, and R. Rosales. Resonantly interacting weakly nonlinear hyperbolic waves ii: several space variable. Studies in Applied Mathematics, 75:187-226, 1986.

[15] J. Joly, G. Metivier, and J. Rauch. Resonant one dimensional nonlinear geometric optics. Journal of Functional Analysis, Jan 1993.

[16] J. Joly, G. Metivier, and J. Rauch. Global solvability of the anharmonic oscillator model from nonlinear optics. SIAM Journal on Mathematical Analysis, Jan 1996.

[17] D. Ketcheson. High Order Strong Stability Preserving Time Integrators and Numerical Wave Propagation Methods for Hyperbolic PDEs. PhD thesis, University of Washington, 2009.

[18] P. Kinsler. Limits of the unidirectional pulse propagation approximation. Journal of the Optical Society of America B: Optical Physics, 24(9):2363-2368, Jan 2007.

[19] P. Kinsler, S. Radnor, J. Tyrrell, and G. New. Optical carrier wave shocking: Detection and dispersion. Physical Review E - Statistical, Nonlinear, and Soft Matter Physics, 75(6), Jan 2007.

[20] S. Klainerman and A. Majda. Formation of singularities for wave equations including the nonlinear vibrating string. Comm. Pure Appl. Math, 33(3):241-263, 1980.

[21] D. Lannes. Dispersive effects for nonlinear geometrical optics with rectification. Asymptotic Anal, 18(1-2):111-146, Jan 1998.

[22] P. Lax. Development of singularities of solutions of nonlinear hyperbolic partial differential equations. Journal of Mathematical Physics, 5:611, 1964.

[23] P. Lax. Hyperbolic Partial Differential Equations. American Mathematical Society, 2006.

[24] R. LeVeque. CLAWPACK. http://www.amath.washington.edu/ claw/.

[25] R. LeVeque. Finite volume methods for hyperbolic problems. Cambridge university press, 2002.

[26] R. LeVeque and D. Yong. Solitary waves in layered nonlinear media. SIAM Journal on Applied Mathematics, pages 1539$1560,2003$.

[27] T. Liu. The Riemann problem for general $2 \times 2$ conservation laws. Transactions of the American Mathematical Society, pages $89-112,1974$. 
[28] T. Liu. The Riemann problem for general systems of conservation laws. J. Differential Equations, 18(1):218-234, 1975.

[29] T. Liu. Hyperbolic and viscous conservation laws. Society for Industrial Mathematics, 2000.

[30] A. Majda and R. Rosales. Resonantly interacting weakly nonlinear hyperbolic waves i: a single space variable. Studies in Applied Mathematics, 71:149-179, 1984.

[31] A. J. Majda, R. Rosales, E. G. Tabak, and C. Turner. Interaction of large-scale equatorial waves and dispersion of kelvin waves through topographic resonances. Journal of the Atmospheric Sciences, 56:24, 1999.

[32] S. Müller and A. Voss. The riemann problem for the euler equations with nonconvex and nonsmooth equation of state: construction of wave curves. SIAM Journal on Scientific Computing, 28(2):651-681, 2006.

[33] S. Müller and A. Voss. The Riemann problem for the Euler equations with nonconvex and nonsmooth equation of state: construction of wave curves. SIAM Journal on Scientific Computing, 28:651, 2006.

[34] D. Pelinovsky and G. Schneider. Justification of the coupled-mode approximation for a nonlinear elliptic problem with a periodic potential. Applicable Analysis, 86(8):1017-1036, 2007.

[35] D. Pelinovsky and G. Schneider. Moving gap solitons in periodic potentials. Mathematical Methods in the Applied Sciences, 31(14):1739-1760, 2008.

[36] D. Pelinovsky, G. Simpson, and M. Weinstein. Solitons in the nonlinear maxwell equations. In preparation.

[37] J. Ranka, R. Windeler, and A. Stentz. Optical properties of high-delta air-silica microstructure optical fibers. Optics letters, 25(11):796-798, Jan 2000.

[38] M. Reed and B. Simon. Methods of Modern Mathematical Physics IV. Analysis of Operators. Academic Press, 1978.

[39] G. Rosen. Electromagnetic shocks and the self-annihilation of intense linearly polarized radiation in an ideal dielectric material. Physical Review, 139(2A):A539-A543, Jan 1965.

[40] G. Schneider. Nonlinear coupled mode dynamics in hyperbolic and parabolic periodically structured spatially extended systems. Asymptotic Analysis, 28(2):163-180, 2001.

[41] G. Schneider and H. Uecker. Existence and stability of modulating pulse solutions in Maxwell's equations describing nonlinear optics. Zeitschrift für Angewandte Mathematik und Physik (ZAMP), 54(4):677-712, 2003.

[42] G. Simpson and M. Weinstein. Nonlinear geometrical optics and quadratically nonlinear periodic media. Unpublished Notes, 2010.

[43] D. Sjoberg. On uniqueness and continuity for the quasi-linear, bianisotropic Maxwell equations, using an entropy condition. Progress In Electromagnetics Research, pages 317-339, 2007.

[44] J. Smoller. Shock waves and reaction-diffusion equations. Springer, 1994.

[45] A. Soffer and M. Weinstein. Time dependent resonance theory. Geometric and Functional Analysis (GAFA), 1998.

[46] R. Tasgal, Y. Band, and B. Malomed. Gap solitons in a medium with third-harmonic generation. Physical Review E Statistical, Nonlinear, and Soft Matter Physics, 72(1):1-10, Jan 2005.

[47] B. Wendroff. The Riemann problem for materials with nonconvex equations of state. I. Isentropic flow. J. Math. Anal. Appl, 38:454-466, 1972 .

[48] B. Wendroff. The Riemann problem for materials with nonconvex equations of state. II. General flow. J. Math. Anal. Appl, 38:640-658, 1972.

[49] P. Wesseling and D. Van Der Heul. Uniformly effective numerical methods for hyperbolic systems. Computing, 66(3):249$267,2001$.

[50] P. Wesseling, D. van der Heul, and C. Vuik. Unified methods for computing compressible and incompressible flows. In Proceedings of ECCOMAS, pages 11-14, 2000.

\section{Appendix A. The NLCME Soliton}

Using the notation of [11], the NLCME soliton solution of (2.9) is given by:

$$
\begin{gathered}
\mathcal{E}^{+}(Z, T)=s \alpha e^{\mathrm{i} \eta} \sqrt{\left|\frac{N_{2}}{2 \Gamma}\right| \frac{1}{\Delta} \sin \delta e^{\mathrm{i} s \sigma} \operatorname{sech}(\theta-\mathrm{i} s \delta / 2)} \\
\mathcal{E}^{-}(Z, T)=-\alpha e^{\mathrm{i} \eta} \sqrt{\left|\frac{N_{2}}{2 \Gamma}\right|} \Delta \sin \delta e^{\mathrm{i} s \sigma} \operatorname{sech}(\theta+\mathrm{i} s \delta / 2) \\
\theta=\gamma N_{2} \sin \delta(Z-v T), \quad \sigma=\gamma N_{2} \cos \delta(v Z-T) \\
e^{\mathrm{i} \eta}=\left(-\frac{e^{2 \theta}+e^{-\mathrm{i} s \delta}}{e^{2 \theta}+e^{\mathrm{i} s \delta}}\right)^{2 v /\left(3-v^{2}\right)} \\
\gamma=1 / \sqrt{1-v^{2}}, \quad \Delta=\left(\frac{1-v}{1+v}\right)^{1 / 4} \\
s=\operatorname{sign}\left(N_{2} \Gamma\right), \quad \alpha=\sqrt{\frac{2\left(1-v^{2}\right)}{3-v^{2}}}, \quad \tilde{\kappa}=\kappa N_{2}
\end{gathered}
$$

We assume that $N_{2} \in \mathbb{R}$. There are two free parameters, $|v|<1$ and $\delta \in \mathbb{R}$. 


\section{Appendix B. Simulating the Nonlinear Maxwell Equations}

In vector notation, the rescaled Maxwell system, 3.2 , and constitutive law, $[3.3$, are expressed as

$$
\begin{gathered}
\partial_{t}\left(\begin{array}{c}
D \\
B
\end{array}\right)+\partial_{z}\left(\begin{array}{c}
-B \\
-E(D, z)
\end{array}\right)=0 \\
\partial_{t} \mathbf{v}+\partial_{z} \mathbf{f}(\mathbf{v}, z)=0 .
\end{gathered}
$$

To simulate this system of conservation laws, we employ a shock capturing finite volume scheme with the CLAWPACK software, 24, 25. Furthermore, we employ the $f$-wave method to accommodate the spatially varying flux function, $2,25,26$.

To use finite volume methods we must provide the algorithm with a, possibly approximate, solution of the Riemman problem. This introduces a subtlety as our system has a non-convex flux function. Non-convex fluxes lead to interesting waves, including rightward (or leftward) traveling rarefaction and shockwaves that are "glued" together. Such waves, sometimes called compound or composite waves, were discussed in 27, 28, 47, 48 and more recently in 33, 49,50. Examples are also give in the texts 25, 44].

B.1. Finite Volume Methods for Maxwell. In finite volume numerical methods, at each time step, we must solve a Riemann problem between adjacent grid cells:

$$
\begin{aligned}
& \mathbf{v}_{t}+\mathbf{f}\left(\mathbf{v} ; z_{j}\right)_{z}=0 \text { for } z_{j-1 / 2}<z<z_{j+1 / 2}, \\
& \mathbf{v}_{t}+\mathbf{f}\left(\mathbf{v} ; z_{j+1}\right)_{z}=0 \text { for } z_{j+1 / 2}<z<z_{j+3 / 2} \text {, } \\
& \mathbf{v}\left(z, t=t^{n}\right)= \begin{cases}\mathbf{v}_{j}^{n} & \text { for } z_{j-1 / 2}<z<z_{j+1 / 2}, \\
\mathbf{v}_{j+1}^{n} & \text { for } z_{j+1 / 2}<z<z_{j+3 / 2} .\end{cases}
\end{aligned}
$$

$z_{j+1 / 2}$ is the interface between the cell centered at $z_{j}$ and the cell centered at $z_{j+1 / 2}$. The fluxes are assumed to be constant in $z$ within each computational cell. We aim to provide an exact solution to the Riemann problem, in contrast to an approximate solutions such as the Roe average.

In the next few sections, we adopt the notation

$$
\begin{aligned}
& \mathbf{v}_{t}+\mathbf{f}_{l}(\mathbf{v})_{z}=0 \quad \text { for } z<0 \\
& \mathbf{v}_{t}+\mathbf{f}_{r}(\mathbf{v})_{z}=0 \text { for } z>0 \\
& \mathbf{v}(z, 0)= \begin{cases}\mathbf{v}_{l} & \text { for } z<0 \\
\mathbf{v}_{r} & \text { for } z>0\end{cases}
\end{aligned}
$$

where $\mathbf{f}_{l}(\mathbf{v})=\mathbf{f}\left(\mathbf{v} ; z_{j}\right), \mathbf{f}_{r}(\mathbf{v})=\mathbf{f}\left(\mathbf{v} ; z_{j+1}\right)$, and we take $z_{j+1 / 2}=0$.

Given any point $\mathbf{v}$ in $(D, B)$ phase space, we construct two, entropy condition (specified below) satisfying, manifolds $\mathcal{W}_{1}(\mathbf{v})$ and $\mathcal{W}_{2}(\mathbf{v})$. These define the locus of points that can be joined to $\mathbf{v}$ by a left-going wave in the former case and a right-going wave in the latter case. We parameterize them in the $D$ component. Given a state $\mathbf{v}_{0}, W_{j}\left(D ; \mathbf{v}_{0}\right)$ is the parametric curve such that:

$$
\left\{\left(\begin{array}{c}
D \\
W_{j}\left(D ; \mathbf{v}_{0}\right)
\end{array}\right), \quad \text { for } D \in \mathbb{R}\right\}=\mathcal{W}_{j}\left(\mathbf{v}_{0}\right) \text { for } j=1,2
$$

Were the medium homogeneous, solving the Riemann corresponds to finding the state $\mathbf{v}_{\star}$ that is the unique point in $\mathcal{W}_{1}\left(\mathbf{v}_{l}\right) \cap \mathcal{W}_{2}\left(\mathbf{v}_{r}\right)$. In terms of the parametric curves, this point solves the equation:

$$
W_{2}\left(D_{r} ;\left(\begin{array}{c}
D_{\star} \\
W_{1}\left(D_{\star} ; \mathbf{v}_{l}\right)
\end{array}\right)\right)=B_{r}
$$

As the medium is not homogeneous, we match the flux at the interface. We seek $\mathbf{v}_{\star}^{l}$ and $\mathbf{v}_{\star}^{r}$ such that:

$$
\begin{aligned}
W_{1}\left(D_{l}^{\star} ; \mathbf{v}_{l}\right) & =B_{l}^{\star} \\
\mathbf{f}_{l}\left(\mathbf{v}_{l}^{\star}\right) & =\mathbf{f}_{r}\left(\mathbf{v}_{r}^{\star}\right) \\
W_{2}\left(D_{r} ; \mathbf{v}_{r}^{\star}\right) & =B_{r}
\end{aligned}
$$

$\mathbf{v}_{l}^{\star}$ is the the entropy satisfying state immediately to the left of the interface and $\mathbf{v}_{r}^{\star}$ is the entropy satisfying state immediately to the right of the interface. 
For this problem, the flux matching condition is:

$$
\begin{aligned}
E\left(D_{l}^{\star} ; z_{l}\right) & =E\left(D_{r}^{\star} ; z_{r}\right) \\
B_{l}^{\star} & =B_{r}^{\star}
\end{aligned}
$$

Defining transfer function, $\mathcal{T}$, that, given $z_{l}, z_{r}$ and a left state $D_{l}^{\star}$, the flux matched displacement is:

$$
\mathcal{T}\left(D_{l}^{\star} ; z_{l}, z_{r}\right)=D_{r}^{\star}
$$

With this function, B.5 becomes

$$
W_{2}\left(D_{r} ;\left(\begin{array}{c}
\mathcal{T}\left(D_{l}^{\star} ; z_{l} ; z_{r}\right) \\
W_{1}\left(D_{l}^{\star} ; \mathbf{v}_{l}\right)
\end{array}\right)\right)=B_{r}
$$

$D_{l}^{\star}$ is the unknown. Once we have this value, we recover $E^{\star}$ and $B^{\star}$ allowing us to compute the fluxes. Subject to the specification of the phase space functions $W_{j}$ are specified, this is a one-dimensional root finding problem.

B.2. Non-Convex Fluxes and the Entropy Condition. It remains to specify the manifolds $\mathcal{W}_{j}$. This requires an additional, non-trivial, assumption on an entropy condition. While such a condition is readily apparent in gas dynamics and elasticity, the appropriate condition for Maxwell is non-obvious.

In this work, we employ a diffusive entropy condition, akin to that found in gas dynamics. This was suggested by Sjöberg [43, as part of an entropy-flux pair involving the Poynting vector. This is also a physically consistent, as many dielectrics absorb the higher harmonics that would appear as the wave began to shock.

In constructing the entropy satisfying $W_{j}$ functions, we closely follow $27,28,32,48$ and particularly the $p$-system example in 47 . Graphically, the $W_{j}$ functions can be constructed by tracing an appropriate convex hull of $E(D ; z)$. Shock waves occur when points are joined by chords, rarefaction waves when points are joined along $E(D ; z)$, and composite waves when convex curve is a combination.

Throughout this section we suppress the $z$ argument, and $E^{\prime}(D)=\partial_{D} E(D, z)$.

Since the flux function is no longer uniformly convex, the Lax entropy condition may not be appropriate. Instead, Liu entropy condition 27,32 may apply. Recall, that if

$$
\sigma\left(\mathbf{v}_{0}, \mathbf{v}\right) \equiv \frac{-E(D)+E\left(D_{0}\right)}{B-B_{0}}
$$

then:

- A shock joining $\mathbf{v}_{0}$ to $\mathbf{v}_{1}$ satisfies the Lax entropy condition if the system is convex and either

$$
\begin{array}{ll}
\lambda_{1}\left(\mathbf{v}_{1}\right)<\sigma<\lambda_{1}\left(\mathbf{v}_{0}\right), & \sigma<0 \\
\lambda_{2}\left(\mathbf{v}_{1}\right)<\sigma<\lambda_{2}\left(\mathbf{v}_{0}\right), & \sigma>0
\end{array}
$$

where $\lambda_{1}<0<\lambda_{2}$ are the eigenvalues of

$$
\left(\begin{array}{cc}
0 & -1 \\
-E^{\prime}(D) & 0
\end{array}\right)
$$

- A shock joining $\mathbf{v}_{0}$ to $\mathbf{v}_{1}$ satisfies the Liu entropy condition if

$$
\sigma\left(\mathbf{v}_{0}, \mathbf{v}_{1}\right) \leq \sigma\left(\mathbf{v}_{0}, \tilde{\mathbf{v}}\right)
$$

for all points $\tilde{\mathbf{v}}$ between the two points along the shock curve in phase space.

B.2.1. Left Traveling Waves. Given the state $\mathbf{u}_{0}=\left(D_{0}, B_{0}\right)^{T}$, we construct $W_{1}\left(D ; \mathbf{u}_{0}\right)$. Since we have an inflection point in $E(D)$ at $D=0$, we dissect all the possible configurations of $D_{0}, D$ and the inflection point. Let $D_{1}$ be the value of $D$ at which the line tangent to $\left(D_{1}, E\left(D_{1}\right)\right)$ intercepts $\left(D_{0}, E\left(D_{0}\right)\right)$.

First, suppose that $D<D_{0}<0$, as in Figure 15(a). In this region, there is no difficulty applying the Lax entropy condition (B.10); there is no shock as

$$
\lambda_{1}(D)=-\sqrt{E^{\prime}(D)}<\lambda_{1}\left(D_{0}\right)=-\sqrt{E^{\prime}\left(D_{0}\right)}<0
$$

Consequently,

$$
B=B_{0}+\int_{\substack{D_{0} \\ 27}}^{D} \sqrt{E^{\prime}(s)} d s
$$




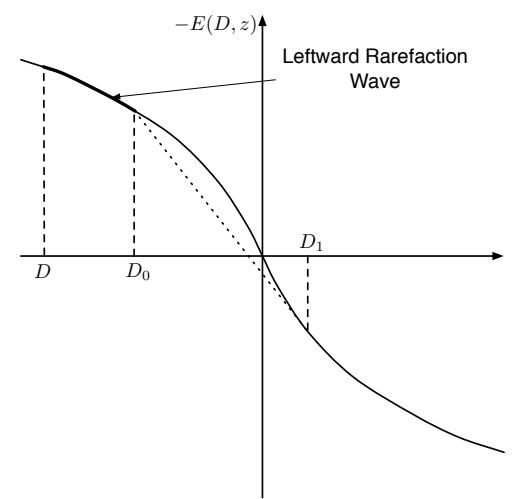

(a) $D<D_{0}$

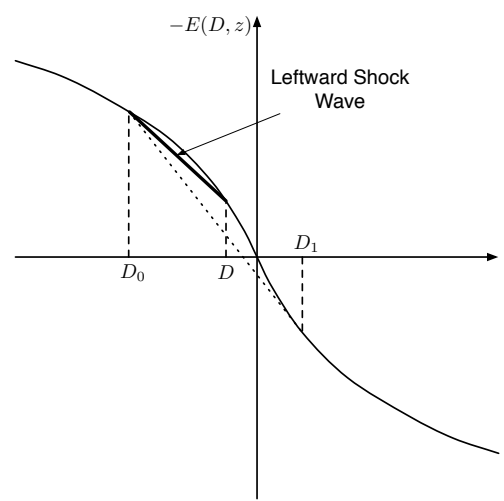

(b) $D_{0}<D<D_{1}$

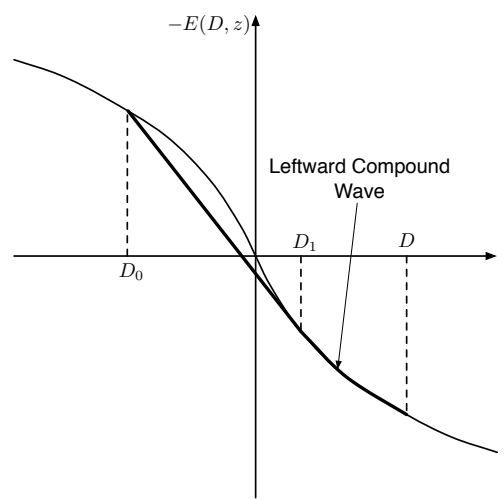

(c) $D_{0}<D_{1}<D$

FIGURE 15. Entropy satisfying leftward traveling waves when $D_{0}<0$.

Still assuming that $D_{0}<0$, if $D_{0}<D<D_{1}$ the Lax condition continues to apply. $D_{0}$ and $D$ will satisfy (B.10) and we can join the two with a shock, as in Figure 15(b)

$$
B=B_{0}+\sqrt{\left|E(D)-E_{0}\right|\left|D-D_{0}\right|}
$$

Once $D>D_{1}$, the solution changes. It is no longer appropriate to apply the Lax condition as we lose convexity here. Applying the Liu condition, (B.11), we see that there is no longer a shock. Indeed, we can compute that were there shock solutions,

$$
\begin{gathered}
\sigma\left(\mathbf{v}_{0}, \mathbf{v}\right)=\frac{-E+E_{0}}{B-B_{0}}=-\sqrt{\left|\frac{E-E_{0}}{D-D_{0}}\right|} \\
\sigma\left(\mathbf{v}_{0}, \mathbf{v}_{1}\right)=\frac{-E_{1}+E_{0}}{B_{1}-B_{0}}=-\sqrt{\left|\frac{E_{1}-E_{0}}{D_{1}-D_{0}}\right|}
\end{gathered}
$$

Examining Figure 15(c)

$$
\frac{E_{1}-E_{0}}{D_{1}-D_{0}}<\frac{E-E_{0}}{D-D_{0}}<0
$$

implying

$$
\sigma\left(\mathbf{v}_{0}, \mathbf{v}_{1}\right)<\sigma\left(\mathbf{v}_{0}, \mathbf{v}\right)
$$

which violates (B.11).

As a shock fails to connect the two states, we resort to joining the states by a compound wave. The solution is a shock from $D_{0}$ to $D_{1}$ which continues into a rarefaction wave from $D_{1}$ to $D$. Thus,

$$
B=B_{0}+\sqrt{\left|E\left(D_{1}\right)-E_{0}\right|\left|D_{1}-D_{0}\right|}+\int_{D_{1}}^{D} \sqrt{E^{\prime}(s)} d s
$$

At $D_{0}=0$ the system is convex yielding leftward traveling rarefaction waves for all values of $D$ :

$$
B=B_{0}+\int_{D_{0}}^{D} \sqrt{E^{\prime}(s)} d s
$$

For $D_{0}>0$, There are again several cases. For $D<D_{1}$, we have the compound wave again, as in Figure $16(\mathrm{a})$.

$$
B=B_{0}-\sqrt{\left|E\left(D_{1}\right)-E_{0}\right|\left|D_{1}-D_{0}\right|}+\int_{D_{1}}^{D} \sqrt{E^{\prime}(s)} d s
$$

As $D$ increases in value and $D_{1}<D<D_{0}$, we have a shock solution

$$
B=B_{0}-\sqrt{\left|E(D)-E_{0}\right|\left|D-D_{0}\right|}
$$




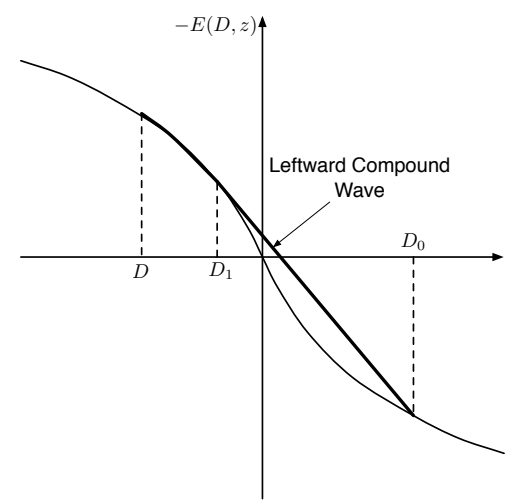

(a) $D<D_{1}<D_{0}$

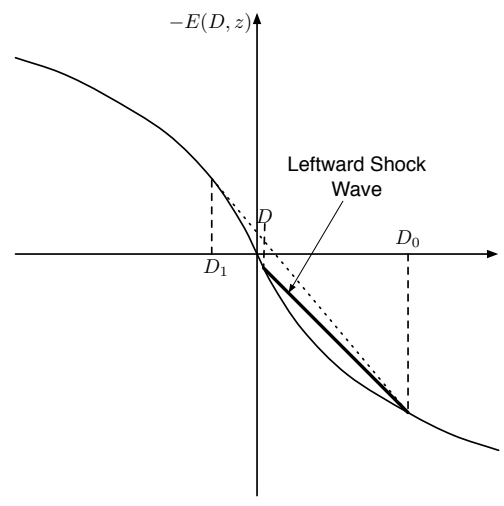

(b) $D_{1}<D<D_{0}$

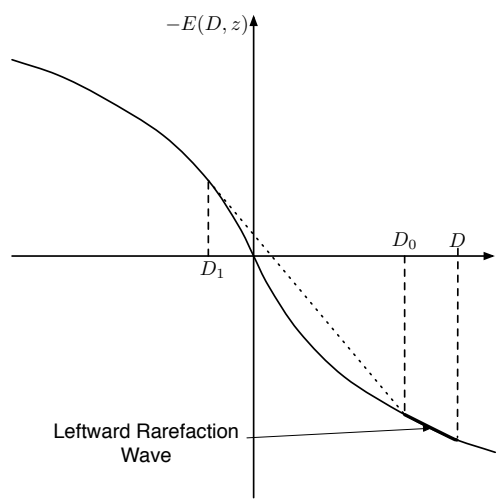

(c) $D_{0}<D$

FiguRE 16. Entropy satisfying leftward traveling waves when $D_{0}>0$.

See Figure 16(b) Lastly, for $D>D_{0}$, we get a leftward traveling rarefaction:

$$
B=B_{0}+\int_{D_{0}}^{D} \sqrt{E^{\prime}(s)} d s
$$

B.2.2. Right Traveling Waves. For right traveling waves, the structure is similar. Given our point $D_{0}$, let $\left(D_{2}, E_{2}\right)$ be the point intercepted by the line tangent to $\left(D_{0}, E\left(D_{0}\right)\right)$.

Again, we first treat $D_{0}<0$. The different cases are diagrammed in Figure 17. For $D<D_{0}<0$, there is a shock,

$$
B=B_{0}+\sqrt{\left|E(D)-E_{0}\right|\left|D-D_{0}\right|}
$$

For $D_{0}<D<0$, this changes to a rarefaction wave,

$$
B=B_{0}-\int_{D_{0}}^{D} \sqrt{E^{\prime}(s)} d s .
$$

Crossing the inflection point, $0<D<D_{2}$, it becomes a compound wave which rarefacts to the point $D_{\star}$ followed by a shock,

$$
B=B_{0}-\int_{D_{0}}^{D_{\star}} \sqrt{E^{\prime}(s)} d s-\sqrt{\left|E(D)-E_{\star}\right|\left|D-D_{\star}\right|} .
$$

$D_{\star}$ is the point $\left(D_{\star}, E_{\star}\right)$ on the curve whose tangent intercepts $(D, E)$. Past $D_{2}$, the compound wave reduces to a shock, as the system now satisfies B.11,

$$
B=B_{0}-\sqrt{\left|E(D)-E_{0}\right|\left|D-D_{0}\right|} .
$$

For $D_{0}=0$, we have a shock in both directions,

$$
B=B_{0}-\operatorname{sign}(D) \sqrt{\left|E(D)-E_{0}\right|\left|D-D_{0}\right|} .
$$

For $D_{0}>0$, again, we must consider the different positions of $D$ relative to the other points. These cases appear in Figure 18. If $D<D_{2}<0$, there is the shock solution satisfying (B.11),

$$
B=B_{0}+\sqrt{\left|E(D)-E_{0}\right|\left|D-D_{0}\right|} .
$$

For $D_{2}<D<0$, this becomes a compound wave,

$$
B=B_{0}-\int_{D_{0}}^{D_{\star}} \sqrt{E^{\prime}(s)} d s+\sqrt{\left|E(D)-E_{\star}\right|\left|D-D_{\star}\right|}
$$

$D_{\star}$ is again the point on the curve whose tangent intercepts $(D, E(D))$. For $0<D<D_{0}$, this becomes a purely rarefactory wave,

$$
B=B_{0}-\int_{D_{0}}^{D} \sqrt{E^{\prime}(s)} d s
$$




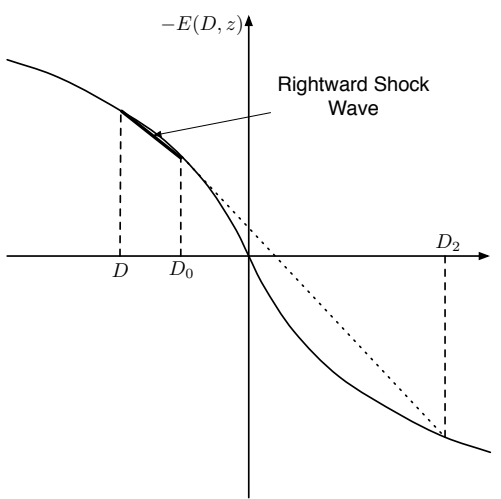

(a) $D<D_{0}$

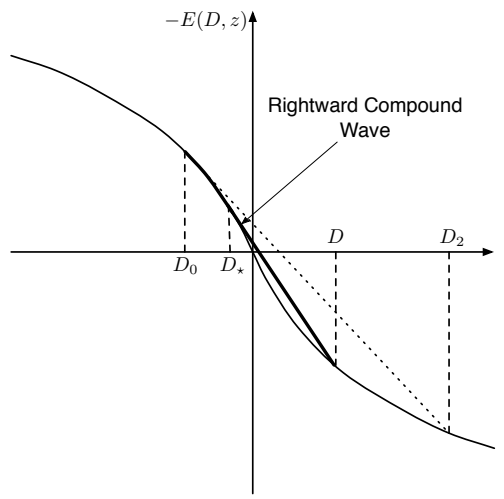

(c) $0<D<D_{2}$

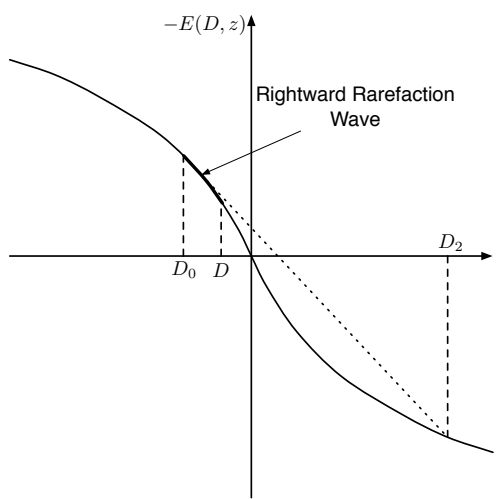

(b) $D_{0}<D<0$

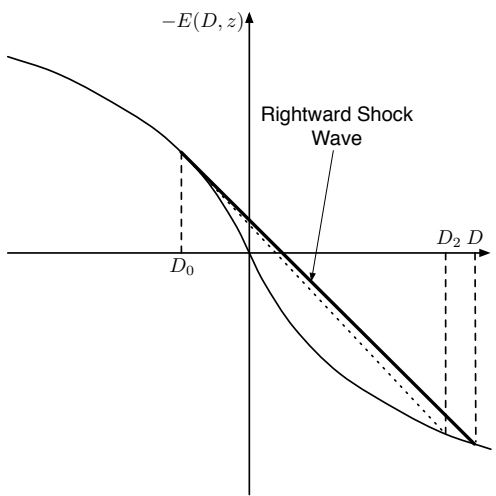

(d) $D_{2}<D$

FIGURE 17. Entropy satisfying rightward traveling waves when $D_{0}<0$.

Finally, for $D>D_{0}$, we again have a shock,

$$
B=B_{0}-\sqrt{\left|E(D)-E_{0}\right|\left|D-D_{0}\right|} .
$$

E-mail address: simpson@math.toronto.edu

Mathematics Department, University of Toronto, Toronto, Ontario, Canada

E-mail address: miw2103@columbia.edu

Applied Mathematics Department, Columbia University, New York City, Ny 10027, USA 


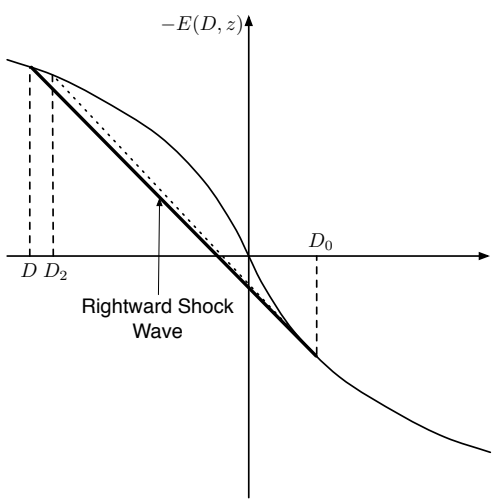

(a) $D<D_{2}$

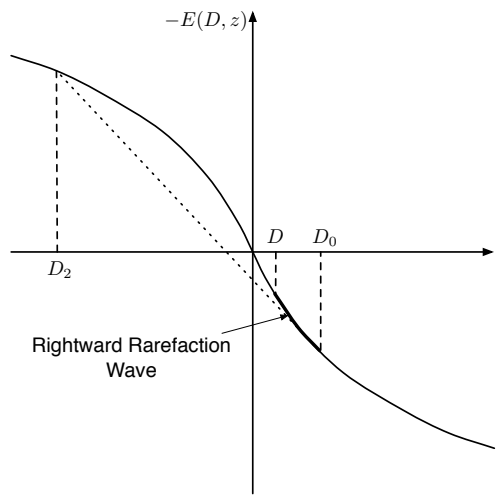

(c) $0<D<D_{0}$

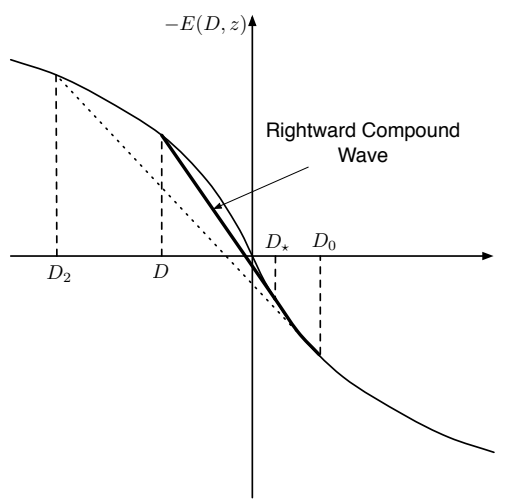

(b) $D_{2}<D<0$

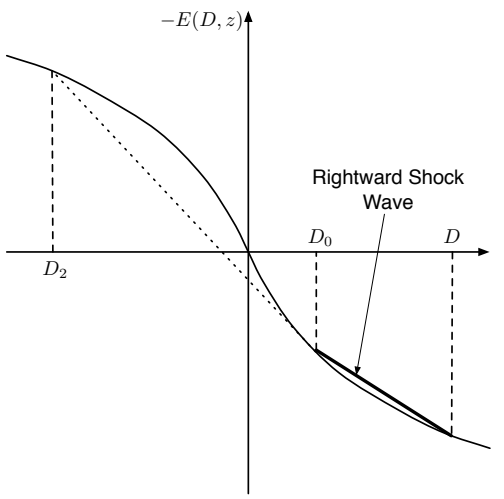

(d) $D_{0}<D$

FiguRE 18. Entropy satisfying rightward traveling waves when $D_{0}>0$. 